\title{
The LS-STAG immersed boundary/cut-cell method for non-Newtonian flows in 3D extruded geometries
}

\author{
F. Nikfarjam, Y. Cheny, O. Botella* \\ LEMTA - Université de Lorraine- 2 avenue de la Forêt de Haye, TSA 60604 - 54518 Vandouvre CEDEX, France
}

\begin{abstract}
The LS-STAG method is an immersed boundary/cut-cell method for viscous incompressible flows based on the staggered MAC arrangement for Cartesian grids, where the irregular boundary is sharply represented by its level-set function, results in a significant gain in computer resources (wall time, memory usage) compared to commercial body-fitted CFD codes. The 2D version of LS-STAG method is now well-established (Y. Cheny \& O. Botella, J. Comput. Phys. Vol. 229, 1043-1076, 2010), and this paper presents its extension to 3D geometries with translational symmetry in the $z$ direction (hereinafter called 3D extruded configurations). This intermediate step towards the fully 3D implementation can be applied to a wide variety of canonical flows and will be regarded as the keystone for the full 3D solver, since both discretization and implementation issues on distributed memory machines are tackled at this stage of development. The LS-STAG method is then applied to various Newtonian and non-Newtonian flows in 3D extruded geometries (axisymmetric pipe, circular cylinder, duct with an abrupt expansion) for which benchmark results and experimental data are available. The purpose of these investigations are (a) to investigate the formal order of accuracy of the LS-STAG method, (b) to asses the versatility of method for flow applications at various regimes (Newtonian and shear-thinning fluids, steady and unsteady laminar to turbulent flows) (c) to compare its performance with well-established numerical methods (body-fitted and immersed boundary methods).
\end{abstract}

Keywords: Computational fluid dynamics (CFD), Non-Newtonian fluids, Immersed boundary methods, Cut-cell methods, High-performance computing (HPC).

\section{Introduction}

Advanced computational fluid dynamics (CFD) applications concern flows in highly irregular three-dimensional geometries, whose process of grid generation, from the early stage of CAD design to final volume meshing, is very time-consuming and requires highly trained users. Typical unstructured body-conformal meshes are made of elements (cells of hexahedral, tetrahedral shape, ...) with irregular connectivity, which makes the solution algorithms extremely memory and CPU intensive. Moreover, it is difficult to assess the quality of the grid (smooth, undistorted cells properly clustered at the wall, ...), which may negatively impact the convergence and accuracy of the numerical solution. In contrast, cut-cell/immersed boundary CFD codes (see [1] for a review) are based on Cartesian methods which are extremely robust and optimized, and thus requires only a fraction of the computer resources of an unstructured code. Cartesian grid generation is greatly simplified compared to the body-conformal approach, and the grid regularity and quality is not significantly impacted by the complexity of the geometry. Moreover, the computation of flows with moving boundaries can be performed on fixed grids, without the domain remeshing which is required by body-conformal methods.

\footnotetext{
* Corresponding author

Email address: olivier.botella@univ-lorraine.fr (O. Botella)
} 
The major difficulty of traditional IB methods is the treatment of the cut-cells (cells of irregular shape which are formed by the intersection of the Cartesian mesh with the immersed boundary). Classical IB methods such as the momentum forcing method 11 discard the discretization of flow equations in the cut-cells, and use special interpolations instead. Thus, strict conservation of mass is not observed, which leads to non-divergence free velocities or non-physical pressure oscillations in the vicinity of the immersed boundary [2. On the other hand, cut-cell methods aim for discretizing the flow equations in cut-cells by ad hoc treatments, whose major drawback is the loss of the 5-points structure (in 2D) of Cartesian methods. Moreover, these methods rely on cell-merging techniques for which $3 \mathrm{D}$ extension is known to be non-trivial [1]. Therefore, the development of an accurate and cost-effective 3D solver based upon the IB/cut-cell approach is still a challenging task.

In Ref. 3] we have developed an innovative cut-cell discretization for 2D incompressible flows called "LS-STAG" (Level-Set, STAGgered), which is based on the classical MAC method for Cartesian staggered grids, where the irregular boundary is sharply represented by its level-set function. In three basic types of 2D cut-cells (pentagonal, trapezoidal and triangular, see [3]), the discretization is designed such as the global invariants of the flow (total mass, momentum and kinetic energy) are preserved at the discrete level. The LS-STAG discretization in the cut-cells is consistent with the MAC discretization used in Cartesian fluid cells, and has the ability to preserve the 5-point Cartesian structure of the stencil, resulting in a highly computationally efficient method. The LSSTAG method has been successfully applied to Newtonian flows at moderate Reynolds number in fixed and moving geometries [3], pseudoplastic flows [4], and viscoelastic flows [5].

The purpose of this paper is to present a progress report towards a fully 3D LS-STAG code for Newtonian and pseudoplastic liquids, totally optimized for high-performance computing. One of the major difficulty in cut-cell discretization for 3D geometries is the large number of cases to consider: we have enumerated 16 different type of cut-cells in $3 \mathrm{D}$ whereas there are only 3 in 2D. In order to mitigate this issue we first consider building the LS-STAG discretization for 3D configurations with translational symmetry in the $z$ direction, that will be subsequently called 3D extruded geometries. This intermediate step towards the fully 3D implementation can be applied to a wide variety of canonical flows (flow in Taylor-Couette reactors, flow past cylinder of arbitrary cross section shape, abrupt axisymmetric expansion/contraction flow, ...) and will be regarded as the keystone for the full 3D solver, since both discretization and implementation issues on distributed memory machines are tackled at this stage of development. In these geometries, 3D cut-cells are only extruded extensions of pentagonal, trapezoidal and triangular cut-cells in 2D, which enables us to extend the principles of the energy-conserving discretization of Ref. [3] to $3 \mathrm{D}$ : the discretization of the continuity equation, pressure gradient, convective fluxes, normal stresses are direct extension of the 2D LS-STAG formulas. The discretization of velocity gradient components such as $\partial w / \partial x$ and $\partial w / \partial y$, which were absent from the $2 \mathrm{D}$ cases, needs further attention. These terms are tentatively discretized with the formula proposed by van der Heiden et al. [6].

The LS-STAG method is then applied to various Newtonian and non-Newtonian flows in 3D extruded geometries (Taylor-Couette reactor, axisymmetric pipe, circular cylinder, duct with sudden expansion) for which benchmark results and experimental data are available. The purpose of these investigations are (a) to evaluate the formal order of accuracy of the LS-STAG method, (b) to asses the versatility of the method for flow applications at various regimes (Newtonian and shear-thinning fluids, steady and unsteady laminar to turbulent flows) (c) to compare its performance with well-established numerical method (unstructured method, IBM method). First, a grid convergence study is conducted on the Hagen-Poiseuille flow for power-law liquids. A thorough comparison on the accuracy and computational costs (memory usage, CPU time) with respect to a commercial CFD package on unstructured grid (ANSYS FLUENT) is performed on the DFG benchmark for cylinder flow in unsteady laminar regime [7. The ability to perform computation of high Reynolds number flow is addressed on the (weakly) turbulent cylinder flow at $\operatorname{Re}=300$ [8, 9], for which comparison with the results of a well-established IB method [10] is provided. The paper is concluded by studying the flow of shear-thinning fluids in an axisymmetric sudden expansion geometry, for which rheological characterization, experimental flow measurements and FLUENT ${ }^{\circledR}$ 
simulations have been performed in [11].

\section{Governing equations for non-Newtonian flows}

Let $\Omega$ be a 3D computational box domain and $\Gamma$ its surface. The governing equations are the incompressible Navier-Stokes equations for non-Newtonian fluids written in conservative form. The continuity equation reads :

$$
\int_{\Gamma} \boldsymbol{v} \cdot \boldsymbol{n} \mathrm{d} S=0
$$

where $\boldsymbol{v}=(u, v, w)$ is the velocity, and the momentum equations in the $x, y$ and $z$ directions are respectively :

$$
\begin{aligned}
& \rho \frac{\mathrm{d}}{\mathrm{d} t} \int_{\Omega} u \mathrm{~d} V+\rho \int_{\Gamma}(\boldsymbol{v} \cdot \boldsymbol{n}) u \mathrm{~d} S+\int_{\Gamma} p \boldsymbol{e}_{x} \cdot \boldsymbol{n} \mathrm{d} S-\int_{\Gamma} \eta(\dot{\gamma})\left(\dot{\gamma}_{x x} \boldsymbol{e}_{x}+\dot{\gamma}_{x y} \boldsymbol{e}_{y}+\dot{\gamma}_{x z} \boldsymbol{e}_{z}\right) \cdot \boldsymbol{n} \mathrm{d} S=0, \\
& \rho \frac{\mathrm{d}}{\mathrm{d} t} \int_{\Omega} v \mathrm{~d} V+\rho \int_{\Gamma}(\boldsymbol{v} \cdot \boldsymbol{n}) v \mathrm{~d} S+\int_{\Gamma} p \boldsymbol{e}_{y} \cdot \boldsymbol{n} \mathrm{d} S-\int_{\Gamma} \eta(\dot{\gamma})\left(\dot{\gamma}_{y x} \boldsymbol{e}_{x}+\dot{\gamma}_{y y} \boldsymbol{e}_{y}+\dot{\gamma}_{y z} \boldsymbol{e}_{z}\right) \cdot \boldsymbol{n} \mathrm{d} S=0, \\
& \rho \frac{\mathrm{d}}{\mathrm{d} t} \int_{\Omega} w \mathrm{~d} V+\rho \int_{\Gamma}(\boldsymbol{v} \cdot \boldsymbol{n}) w \mathrm{~d} S+\int_{\Gamma} p \boldsymbol{e}_{z} \cdot \boldsymbol{n} \mathrm{d} S-\int_{\Gamma} \eta(\dot{\gamma})\left(\dot{\gamma}_{z x} \boldsymbol{e}_{x}+\dot{\gamma}_{z y} \boldsymbol{e}_{y}+\dot{\gamma}_{z z} \boldsymbol{e}_{z}\right) \cdot \boldsymbol{n} \mathrm{d} S=0
\end{aligned}
$$

where $\rho$ is the fluid density, $p$ is the pressure and $\dot{\gamma}=\left(\nabla \boldsymbol{v}+\nabla \boldsymbol{v}^{T}\right)$ is the rate-of-strain tensor.

In the following two rheological models are employed to describe the shear-dependent dynamic viscosity $\eta(\dot{\gamma})$, where the scalar shear-rate $\dot{\gamma}$ is defined as :

$$
\dot{\gamma}=\sqrt{\frac{1}{2} \dot{\gamma}: \dot{\gamma}}=\sqrt{\left[\dot{\gamma}_{x x}\right]^{2}+\left[\dot{\gamma}_{y y}\right]^{2}+\left[\dot{\gamma}_{z z}\right]^{2}+\left[\dot{\gamma}_{x y}\right]^{2}+\left[\dot{\gamma}_{x z}\right]^{2}+\left[\dot{\gamma}_{y z}\right]^{2}} .
$$

The simplest one is the power-law (Ostwald-de Waele) model :

$$
\eta(\dot{\gamma})=k \dot{\gamma}^{n-1},
$$

where $k$ and $n$ are the consistency and power-law index respectively. Based on the value of the power-law index, the fluid exhibits 3 distinct non-Newtonian behaviors: shear-thinning or pseudoplastic $(n<1)$, Newtonian $(n=1)$ and shear-thickening or dilatant $(n>1)$. The powerlaw model is widely used in engineering applications and its simplicity allows to derive analytical solutions of the Navier-Stokes equations for canonical flows [12]. This rheological model will be used for evaluating the accuracy of the LS-STAG method in Section 6.1. However the powerlaw model describes only approximatively the behavior of a real non-Newtonian fluid and most notably fails to predict the Newtonian plateaus that reaches the viscosity at low $\left(\eta \cong \eta_{0}\right)$ and high $\left(\eta \cong \eta_{\infty}\right)$ shear-rates. These limitations are overcomed by the Cross model :

$$
\eta(\dot{\gamma})=\eta_{\infty}+\frac{\left(\eta_{0}-\eta_{\infty}\right)}{1+(K \dot{\gamma})^{m}},
$$

where $K$ and $m$ are 2 fitting parameters. The Cross parameters can be correlated to the power-law parameters (4) by observing that $k=\eta_{0} K^{-m}$ and $n=1-m$ in the limiting case where $\eta_{0} \gg \eta_{\infty}$, $K \dot{\gamma} \gg 1$ and $\eta_{\infty}$ is small. The Cross model will be used for evaluating the LS-STAG method against experimental results in Sect. 6.3

\section{The LS-STAG mesh for 3D extruded geometries}

We consider a 3D irregular solid domain $\Omega^{\mathrm{ib}}$ which is embedded in the computational domain $\Omega$, such that $\Omega^{\mathrm{f}}=\Omega \backslash \Omega^{\mathrm{ib}}$ represents the fluid domain where the flow equations are to be discretized. To keep track of the irregular boundary $\Gamma^{\mathrm{ib}}$, we employ a signed distance function $\phi(\boldsymbol{x})$ (i.e. , the level-set function [13]) such that $\phi(\boldsymbol{x})$ is negative in the fluid region $\Omega^{\mathrm{f}}, \phi(\boldsymbol{x})$ is positive in the solid region $\Omega^{\mathrm{ib}}$, and such that the boundary $\Gamma^{\mathrm{ib}}$ corresponds to the zero level-set of this function. 


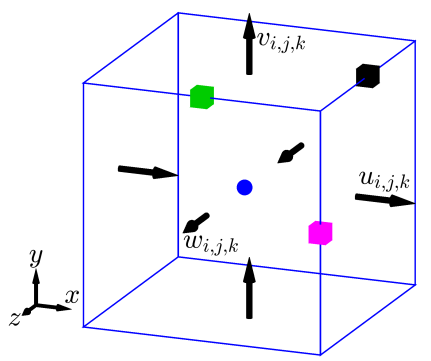

(a) Cartesian cell

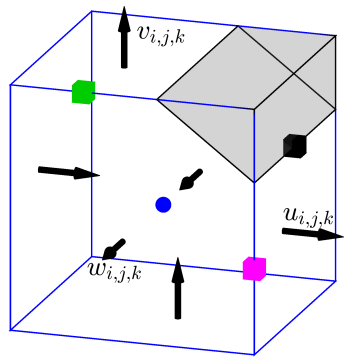

(b) Pentagonal cell

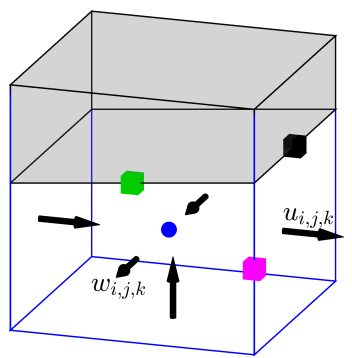

(c) Trapezoidal cell

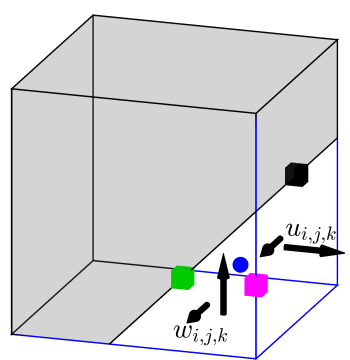

(d) Triangular cell

Figure 1: Four basic types of fluid cells present in the LS-STAG mesh for extruded geometries. Velocity unknowns are located at face centroids, pressure and diagonal components of $\nabla \boldsymbol{v}$ at cell centroids ( ), off-diagonal components of $\nabla \boldsymbol{v}$ at edge midpoints : $\frac{\partial u}{\partial y}, \frac{\partial v}{\partial x}(\mathbf{\square}), \frac{\partial w}{\partial x}, \frac{\partial u}{\partial z}(\square)$ and $\frac{\partial w}{\partial y}, \frac{\partial v}{\partial z}(\square)$.

This leads to the extension of the well-known MAC mesh (see e.g. [14]) that will be subsequently referred to as the $L S-S T A G$ mesh. In the case of irregular geometries extruded in the $z$ direction, the computational cells $\Omega_{i, j, k}$ are either Cartesian cells of size $\Delta x_{i} \times \Delta y_{j} \times \Delta z_{k}$ or cut-cells, whose generic types (pentagonal, trapezoidal and triangular) are depicted in Figure 1 . For all cell types, the velocities unknowns are located at face centers and the pressure $p_{i, j, k}$ is at cell centroids. In the cut-cell shown in Figure 2 the immersed boundary is represented by the rectangular plane $\Gamma_{i, j, k}^{\mathrm{ib}}$ whose extremities are defined by linear interpolation of the level-set function $\phi_{i, j, k}=\phi\left(x_{i}, y_{j}, z_{k}\right)$ at cell corners. This leads to the following decomposition of the surface $\Gamma_{i, j, k}$ of a cut-cell :

$$
\Gamma_{i, j, k}=\Gamma_{i, j, k}^{\mathrm{w}} \cup \Gamma_{i, j, k}^{\mathrm{e}} \cup \Gamma_{i, j, k}^{\mathrm{s}} \cup \Gamma_{i, j, k}^{\mathrm{n}} \cup \Gamma_{i, j, k}^{\mathrm{b}} \cup \Gamma_{i, j, k}^{\mathrm{t}} \cup \Gamma_{i, j, k}^{\mathrm{ib}},
$$

by using the usual compass notations for Cartesian discretizations [15].

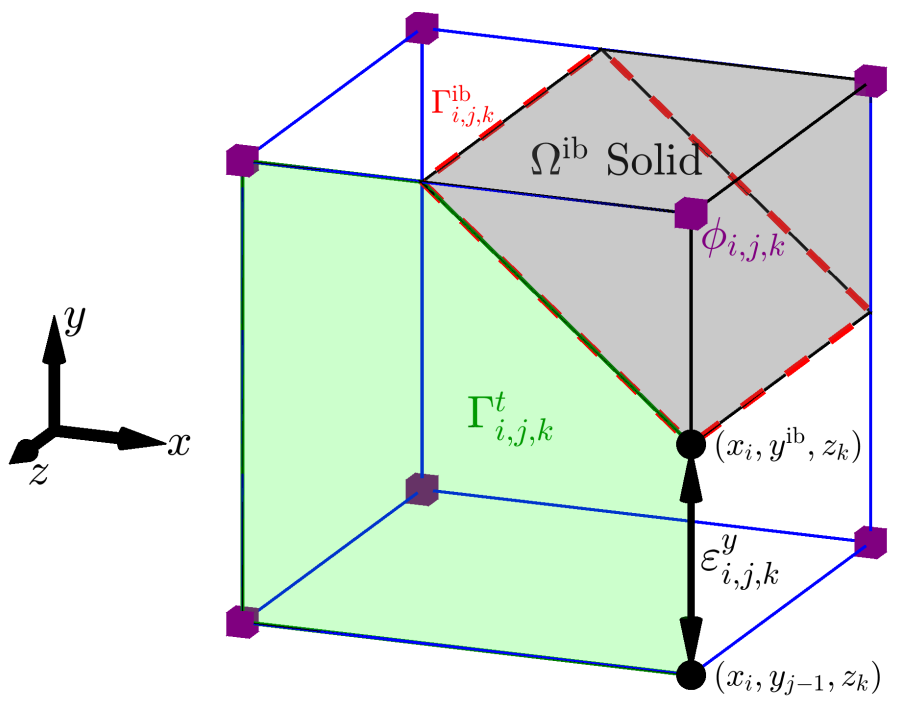

Figure 2: Geometry parameters of pentagonal cut-cell $\Omega_{i, j, k}$. The top elementary fluid face $\Gamma_{i, j, k}^{\mathrm{t}}$ is colored in green, the immersed boundary face $\Gamma_{i, j, k}^{\mathrm{ib}}$ is outlined with red dashed line (--) and the level-set values $\phi_{i, j, k}$ are stored at cell vertices ( $\square$ ).

In [3], the level-set function was employed to obtain analytical expressions of the geometry parameters of 2D cut-cells, such as their face areas and volume. These definitions are now extended to the $3 \mathrm{D}$ case, by calculating first the fluid portion of the cut-cell edges. For the cut-cell 
of Figure 2 the length of Edge $\mathcal{E}_{i, j, k}^{y}=\left\{\left(x_{i}, y, z_{k}\right), y \in\left[y_{j-1}, y^{\mathrm{ib}}\right]\right\}$ is $y^{\mathrm{ib}}-y_{j-1}=\alpha_{i, j, k}^{y} \Delta y_{j}$, where the cell-edge fraction ratio $\alpha_{i, j, k}^{y}$ is computed by linear interpolation of $\phi\left(x_{i}, y, z_{k}\right)$ as :

$$
\alpha_{i, j, k}^{y}=\frac{\phi_{i, j-1, k}}{\phi_{i, j-1, k}-\phi_{i, j, k}},
$$

since $\phi\left(x_{i}, y^{\mathrm{ib}}, z_{k}\right)=0$. Note that cell-edge fraction ratios take values in $[0,1]$ by construction, and due to extrusion $\alpha_{i, j, k-1}^{x}=\alpha_{i, j, k}^{x}, \alpha_{i, j, k-1}^{y}=\alpha_{i, j, k}^{y}$ and $\alpha_{i, j, k}^{z}$ is either 0 or 1 .

The cell-edge fraction ratios are subsequently used to calculate the fluid area of cut-cell faces.

For example, the fluid area of the top face $\Gamma_{i, j, k}^{\mathrm{t}}$ in Figure 2 is $\theta_{i, j, k}^{w} \Delta x_{i} \Delta y_{j}$ where the cell-face fraction ratio is:

$$
\theta_{i, j, k}^{w}=1-\frac{1}{2}\left(1-\alpha_{i, j, k}^{x}\right)\left(1-\alpha_{i, j, k}^{y}\right),
$$

for a pentagonal face. Analytical expressions for trapezoidal and triangular faces (which correspond to volumes in a $2 \mathrm{D}$ mesh) are given in [3]. Finally, the volume $V_{i, j, k}$ of a cut-cell is simply :

$$
V_{i, j, k}=\left(\theta_{i, j, k}^{w} \Delta x_{i} \Delta y_{j}\right) \Delta z_{k} .
$$

since each cut-cell is a prism of base $\Gamma_{i, j, k}^{\mathrm{t}}$ due to extrusion. These geometry parameters will extensively be used to discretize the flow equations in the next sections.

\section{Discretization of the continuity equation}

The starting point of the discretization concerns the continuity equation (1), which is written as the net mass flux through each elementary face of control volume (CV) $\Omega_{i, j, k}$ according to (6) :

$$
\dot{m}^{i, j, k} \equiv-\bar{u}_{i-1, j, k}+\bar{u}_{i, j, k}-\bar{v}_{i, j-1, k}+\bar{v}_{i, j, k}-\bar{w}_{i, j, k-1}+\bar{w}_{i, j, k}+\bar{U}_{i, j, k}^{\mathrm{ib}}=0 .
$$

In the following, we consider homogeneous boundary conditions at the immersed boundary $\Gamma^{\mathrm{ib}}$, so that the mass flux through the immersed boundary $\bar{U}_{i, j, k}^{\mathrm{ib}} \equiv \int_{\Gamma_{i, j, k}^{\mathrm{ib}}} \boldsymbol{v}^{\mathrm{ib}} \cdot \boldsymbol{n}_{i, j, k}^{\mathrm{ib}} \mathrm{d} S$ is zero. The mass fluxes through fluid faces (denoted with a bar) are approximated with midpoint quadrature, yielding for example at the top face :

$$
\bar{w}_{i, j, k} \equiv \int_{\Gamma_{i, j, k}^{\mathrm{t}}} \boldsymbol{v} \cdot \boldsymbol{e}_{z} \mathrm{~d} S \cong \theta_{i, j, k}^{w} \Delta x_{i} \Delta y_{j} w_{i, j, k} .
$$

After similar discretizations at the other faces, the discrete continuity equation reads :

$$
\begin{aligned}
\dot{m}^{i, j, k} \cong & \left(\theta_{i, j, k}^{u} u_{i, j, k}-\theta_{i-1, j, k}^{u} u_{i-1, j, k}\right) \Delta y_{j} \Delta z_{k}+ \\
& \left(\theta_{i, j, k}^{v} v_{i, j, k}-\theta_{i, j-1, k}^{v} v_{i, j-1, k}\right) \Delta x_{i} \Delta z_{k}+ \\
& \left(\theta_{i, j, k}^{w} w_{i, j, k}-\theta_{i, j, k-1}^{w} w_{i, j, k-1}\right) \Delta x_{i} \Delta y_{j}=0
\end{aligned}
$$

and is valid for any type of fluid cells.

\section{Momentum discretization}

Momentum discretization will be illustrated for the $x$-momentum equation $2 \mathrm{a}$ ) in the staggered $\mathrm{CV} \Omega_{i, j, k}^{u}$ represented in Figure 3. This CV is centered around $u_{i, j, k}$ and is formed by halves of neighboring cut-cells $\Omega_{i, j, k}$ and $\Omega_{i+1, j, k}$, such as its volume is $\frac{1}{2} V_{i, j, k}+\frac{1}{2} V_{i+1, j, k}$ and its surface is decomposed as :

$$
\Gamma_{i, j, k}^{u}=\Gamma_{i, j, k}^{u, \mathrm{w}} \cup \Gamma_{i, j, k}^{u, \mathrm{e}} \cup \Gamma_{i, j, k}^{u, \mathrm{~s}} \cup \Gamma_{i, j, k}^{u, \mathrm{ib}} \cup \Gamma_{i, j, k}^{u, \mathrm{~b}} \cup \Gamma_{i, j, k}^{u, \mathrm{t}} .
$$




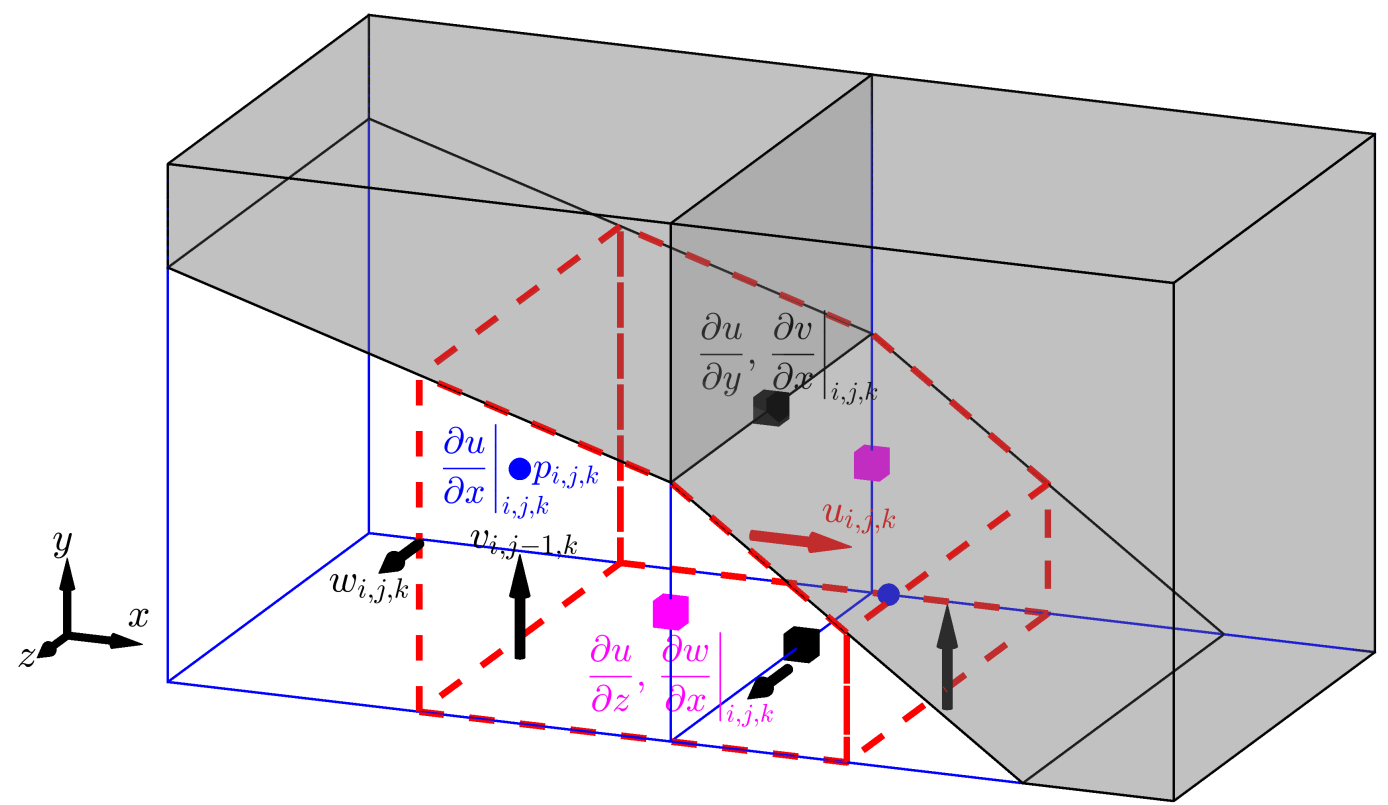

Figure 3: Staggered arrangement of the variables for two adjacent cut-cells on the LS-STAG mesh. CV $\Omega_{i, j, k}^{u} \in$ $\Omega_{i, j, k} \cup \Omega_{i+1, j, k}$ is indicated in red dashed line (--).

\subsection{Basics of the LS-STAG discretization}

As in 2D [3, 4, the key idea of the LS-STAG discretization is to enforce the strict conservation of total mass $\int_{\Omega^{\mathrm{f}}} \nabla \cdot \boldsymbol{v} \mathrm{d} V$, total momentum $\mathbf{P}(t)=\rho \int_{\Omega^{\mathrm{f}}} \boldsymbol{v} \mathrm{d} V$ and total kinetic energy $\mathrm{E}_{\mathrm{c}}(t)=$ $\frac{1}{2} \int_{\Omega^{\mathrm{f}}}|\boldsymbol{v}|^{2} \mathrm{~d} V$ (when viscosity $\eta$ becomes negligible) in each of the half CVs of $\Omega_{i, j, k}^{u}$ independently, such that any combination of half CVs yields a consistent discretization with the aforementioned global conservation properties. This results to a discrete pressure gradient which is dual to the discrete divergence (10), a skew-symmetric discretization for the convective terms, and a viscous stress discretization which is positive definite.

Some of these terms are direct extensions of the 2D formulas: the discrete divergence (10), the discrete pressure gradient which reads in the $x$-direction :

$$
\int_{\Gamma_{i, j, k}^{u}} p \boldsymbol{e}_{x} \cdot \boldsymbol{n} \mathrm{d} S \cong\left[\mathcal{G}^{x} P\right]_{i, j, k}=\theta_{i, j, k}^{u} \Delta y_{j} \Delta z_{k}\left(p_{i+1, j, k}-p_{i, j, k}\right),
$$

and the skew-symmetric discretization of the convective terms, which amounts to using centered approximations with constant weights irrespective of the cell size [14], for example at top face $\Gamma_{i, j, k}^{u, \mathrm{t}}$ :

$$
\int_{\Gamma_{i, j, k}^{u, \mathrm{t}}} u\left(\boldsymbol{v} \cdot \boldsymbol{e}_{z}\right) \mathrm{d} S \cong \frac{u_{i, j, k}+u_{i, j, k+1}}{2} \frac{\bar{w}_{i, j, k}+\bar{w}_{i+1, j, k}}{2} .
$$

These formulas are valid for any type of fluid cells, and in the particular case of Cartesian fluid cells (such that the cell-face fraction ratios are equal to 1), the MAC discretization on non-uniform Cartesian grids of Verstappen \& Veldman [14] is recovered.

In contrast, the discretization of the viscous stresses in extruded cut-cells introduces additional difficulties compared to the $2 \mathrm{D}$ case, which will be discussed in the next sections. 


\subsection{Discretization of the velocity gradient}

We first consider the discretization of the velocity gradient :

$$
\nabla \boldsymbol{v}=\left(\begin{array}{lll}
\frac{\partial u}{\partial x} & \frac{\partial v}{\partial x} & \frac{\partial w}{\partial x} \\
\frac{\partial u}{\partial y} & \frac{\partial v}{\partial y} & \frac{\partial w}{\partial y} \\
\frac{\partial u}{\partial z} & \frac{\partial v}{\partial z} & \frac{\partial w}{\partial z}
\end{array}\right)
$$

which plays an important role in the numerical modeling of the viscous fluxes presented in the next section since it defines the strain-rate tensor $\dot{\gamma}$ and the shear-rate $\dot{\gamma}$ from which the local viscosity is evaluated. Due to the staggering of the velocity unknowns, the diagonal and offdiagonal components of $\nabla \boldsymbol{v}$ are respectively located at centroids and edges of the computational cells (see Figure 1), leading thus to distinct discretization formulas.

The computation of the diagonal components of $\nabla \boldsymbol{v}$ at cell centroids is performed as in the 2D case by enforcing the Green theorem $\int_{\Omega} \nabla \cdot \boldsymbol{v} \mathrm{d} V=\int_{\Gamma} \boldsymbol{v} \cdot \boldsymbol{n} \mathrm{d} S$ at the discrete level [3]. This leads to a unique formula in all types of cut-cells, e.g. for $\partial u / \partial x$ :

$$
\left.\frac{\partial u}{\partial x}\right|_{i, j, k} \cong \frac{\theta_{i, j, k}^{u} u_{i, j, k}-\theta_{i-1, j, k}^{u} u_{i-1, j, k}}{V_{i, j, k} /\left(\Delta z_{k} \Delta y_{j}\right)},
$$

which reduces to a standard two-point formula in Cartesian cells.

An issue related to the 3D extension of the LS-STAG method concerns the computation of the off-diagonal components of $\nabla \boldsymbol{v}$ at the edge midpoints where the orthogonality property of the MAC mesh is lost [16. In the case of extruded geometries, it only concerns terms $\partial w / \partial x$ and $\partial w / \partial y$ in the cut-cells (see Figure 3 where Edge $\mathcal{E}_{i, j, k}^{y}$ and the line connecting $w_{i, j, k}$ and $w_{i+1, j, k}$ is not orthogonal), where the use of a standard two-point finite-difference formula would be inaccurate unless a non-orthogonal correction is taken into account [16. The design of such a scheme, which requires data interpolation and leads to an enlarged stencil near the cut-cells is left to future work. Instead, we employ the discretization of Van der Plas et al. [6] who suggest to compute these terms by analogy with the pressure gradient in $2 \mathrm{D}$ :

$$
\left.\frac{\partial w}{\partial x}\right|_{i, j, k} \cong \frac{\alpha_{i, j, k}^{y} \Delta y_{j}\left(w_{i+1, j, k}-w_{i, j, k}\right)}{\frac{1}{2}\left(\theta_{i+1, j, k}^{w} \Delta x_{i+1} \Delta y_{j}+\theta_{i, j, k}^{w} \Delta x_{i} \Delta y_{j}\right)},
$$

and $\partial w / \partial y$ is computed similarly. The impact of this ad hoc two-point formula on the overall accuracy of the method will be investigated in details in Section 6.1

For other terms standard 2-point formulas are used, such as for $\partial u / \partial y$ at the midpoint of Edge $\mathcal{E}_{i, j-1, k}^{z}$ in Figure 3:

$$
\left.\frac{\partial u}{\partial y}\right|_{i, j-1, k} \cong \frac{u_{i, j, k}-u_{i, j-1, k}}{\widetilde{\Delta y}}, \quad \widetilde{\Delta y}=\frac{1}{2} \alpha_{i, j, k}^{y} \Delta y_{j}+\frac{1}{2} \Delta y_{j-1},
$$

and at the midpoint of solid edge $\mathcal{E}_{i, j, k}^{z}$ :

$$
\left.\frac{\partial u}{\partial y}\right|_{i, j, k} \cong \frac{0-u_{i, j, k}}{\Delta y^{\mathrm{ib}}}, \quad \Delta y^{\mathrm{ib}}=\frac{1}{2} \alpha_{i, j, k}^{y} \Delta y_{j}
$$

for the case of homogeneous boundary conditions. However, for terms such as $\partial v / \partial x$ at the solid boundary, similar formulas cannot be derived since $\theta_{i, j, k}^{v}$ and $\theta_{i+1, j, k}^{v}$ are both null. To address this issue, we have derived a discretization of $\partial v /\left.\partial x\right|_{i, j, k}$ by enforcing that the Schwarz theorem (symmetry of second derivatives) :

$$
\frac{\partial}{\partial y} \frac{\partial v}{\partial x}=\frac{\partial}{\partial x} \frac{\partial v}{\partial y}
$$


holds discretely on cell face $\Gamma_{i, j, k}^{\mathrm{e}}$. Firstly, the LHS of $(21)$ is readily discretized as :

$$
\left.\frac{\partial}{\partial y}\left(\frac{\partial v}{\partial x}\right)\right|_{i, j, k}=\frac{1}{2 \Delta y^{\mathrm{ib}}}\left[\left.\frac{\partial v}{\partial x}\right|_{i, j, k}-\left.\frac{\partial v}{\partial x}\right|_{i, j-1, k}\right]
$$

where $\partial v /\left.\partial x\right|_{i, j-1, k}$ has an expression similar to 19 . Secondly, the RHS of 21 is iscretized by analogy with the pressure gradient (14) since $\partial v / \partial y$ is also located at cell centroids, leading to :

$$
\left.\frac{\partial}{\partial x}\left(\frac{\partial v}{\partial y}\right)\right|_{i, j, k}=\frac{\theta_{i, j, k}^{u} \Delta y_{j} \Delta z_{k}}{\frac{1}{2} V_{i, j, k}+\frac{1}{2} V_{i+1, j, k}}\left[\left.\frac{\partial v}{\partial y}\right|_{i+1, j, k}-\left.\frac{\partial v}{\partial y}\right|_{i, j, k}\right] .
$$

Finally, the identification of 22$]$ with 23 gives the expression of $\partial v /\left.\partial x\right|_{i, j, k}$ :

$$
\left.\frac{\partial v}{\partial x}\right|_{i, j, k}=\left.\frac{\partial v}{\partial x}\right|_{i, j-1, k}+\frac{\theta_{i, j, k}^{u} \Delta y_{j} \Delta z_{k} \Delta y^{\mathrm{ib}}}{\frac{1}{4} V_{i, j, k}+\frac{1}{4} V_{i+1, j, k}}\left[\left.\frac{\partial v}{\partial y}\right|_{i+1, j, k}-\left.\frac{\partial v}{\partial y}\right|_{i, j, k}\right],
$$

which is completed with discretization formulas for $\partial v /\left.\partial y\right|_{i, j, k}$ and $\partial v /\left.\partial y\right|_{i+1, j, k}$ analogous to (17).

\subsection{Discretization of the viscous fluxes}

The discrete velocity gradients are now used for discretizing the viscous fluxes of the $x$ momentum equation $2 \mathrm{al}$ :

$$
F_{u}^{\mathrm{v}}=\int_{\Gamma_{i, j, k}^{u}} \eta(\dot{\gamma}) \dot{\gamma}_{x x} \boldsymbol{e}_{x} \cdot \boldsymbol{n} \mathrm{d} S+\int_{\Gamma_{i, j, k}^{u}} \eta(\dot{\gamma}) \dot{\gamma}_{x y} \boldsymbol{e}_{y} \cdot \boldsymbol{n} \mathrm{d} S+\int_{\Gamma_{i, j, k}^{u}} \eta(\dot{\gamma}) \dot{\gamma}_{x z} \boldsymbol{e}_{z} \cdot \boldsymbol{n} \mathrm{d} S
$$

As in our previous works [3, 4, the normal stress flux is computed by analogy with the pressure gradient (14) :

$$
\int_{\Gamma_{i, j, k}^{u}} \eta(\dot{\gamma}) \dot{\gamma}_{x x} \boldsymbol{e}_{x} \cdot \boldsymbol{n} \mathrm{d} S \cong \theta_{i, j, k}^{u} \Delta y_{j} \Delta z_{k}\left[\left.2 \eta\left(\dot{\gamma}_{\mathrm{e}}\right) \frac{\partial u}{\partial x}\right|_{i+1, j, k}-\left.2 \eta\left(\dot{\gamma}_{\mathrm{w}}\right) \frac{\partial u}{\partial x}\right|_{i, j, k}\right],
$$

and the discretization is completed with Eq. (17).

For the shear stress fluxes, midpoint quadrature is applied to each of the faces of $\Gamma_{i, j, k}^{u}$ (given by (13)), leading to:

$$
\begin{aligned}
& \int_{\Gamma_{i, j, k}^{u, \mathrm{~s}} \cup \Gamma_{i, j, k}^{u, \mathrm{~b}}} \eta(\dot{\gamma}) \dot{\gamma}_{x y} \boldsymbol{e}_{y} \cdot \boldsymbol{n} \mathrm{d} S \cong \Delta S^{\mathrm{ib}} \eta\left(\dot{\gamma}_{\mathrm{n}}\right)\left[\frac{\partial u}{\partial y}+\frac{\partial v}{\partial x}\right]_{i, j, k}-\Delta S^{\mathrm{s}} \eta\left(\dot{\gamma}_{\mathrm{s}}\right)\left[\frac{\partial u}{\partial y}+\frac{\partial v}{\partial x}\right]_{i, j-1, k} \\
& \int_{\Gamma_{i, j, k}^{u, \mathrm{t}} \cup \Gamma_{i, j, k}^{u, \mathrm{~b}}} \eta(\dot{\gamma}) \dot{\gamma}_{x z} \boldsymbol{e}_{z} \cdot \boldsymbol{n} \mathrm{d} S \cong \Delta S^{\mathrm{t}} \eta\left(\dot{\gamma}_{\mathrm{t}}\right)\left[\frac{\partial u}{\partial z}+\frac{\partial w}{\partial x}\right]_{i, j, k}-\Delta S^{\mathrm{b}} \eta\left(\dot{\gamma}_{\mathrm{b}}\right)\left[\frac{\partial u}{\partial z}+\frac{\partial w}{\partial x}\right]_{i, j, k-1}
\end{aligned}
$$

In these formulas, $\Delta S^{\mathrm{ib}}=\frac{1}{2}\left(\Delta x_{i}+\Delta x_{i+1}\right)$ is the projected area of the solid face along the $y$ axis, and the fluid face areas are evaluated by mean of the cell-face fraction ratios, for instance the surface area of $\Gamma^{u, t}$ in Equation $27 \mathrm{~b}$ reads :

$$
\Delta S^{\mathrm{t}}=\frac{1}{2}\left(\theta_{i, j, k}^{w} \Delta x_{i}+\theta_{i+1, j, k}^{w} \Delta x_{i+1}\right) \Delta y_{j},
$$

which ensures local flux conservativity across fluid faces.

For evaluating the non-Newtonian viscosity in (26) and (27), the shear-rate $\dot{\gamma}(3)$ has to be computed at cell centroids for the normal stresses $\left(e . g . \dot{\gamma}_{\mathrm{w}}\right.$ in $\left.(26)\right)$ and at edge midpoints for the shear stresses (e.g. $\dot{\gamma}_{\mathrm{s}}$ in (27)). This is achieved by first computing $\dot{\gamma}$ at cell centroids, for example:

$$
\dot{\gamma}_{\mathrm{w}}=\dot{\gamma}_{i, j, k} \cong \sqrt{\left(\left.\dot{\gamma}_{x x}\right|_{i, j, k} ^{2}+\left.\dot{\gamma}_{y y}\right|_{i, j, k} ^{2}+\left.\dot{\gamma}_{z z}\right|_{i, j, k} ^{2}\right)+\left[\bar{\gamma}_{x y}\right]_{i, j, k}^{2}+\left[\bar{\gamma}_{x z}\right]_{i, j, k}^{2}+\left[\dot{\gamma}_{y z}\right]_{i, j, k}^{2},}
$$


where the off-diagonal terms are interpolated from values at the surrounding edges by arithmetic average $[\because]$. Then, the shear-rate at cell edges is obtained from its neighboring cell-centroid values by using the volume-weighted interpolation first employed in [5] in the framework of viscoelastic flow simulations. For example, the value of $\dot{\gamma}_{\mathrm{s}}$ at Edge $\mathcal{E}_{i, j-1, k}^{z}$ is interpolated as :

$$
\dot{\gamma}_{\mathrm{s}}=\left([\beta V \dot{\gamma}]_{i, j, k}+[\beta V \dot{\gamma}]_{i-1, j, k}+[\beta V \dot{\gamma}]_{i-1, j-1, k}+[\beta V \dot{\gamma}]_{i, j-1, k}\right) / \widetilde{V}_{i, j, k},
$$

where $\widetilde{V}_{i, j, k}$ is the weighted sum of the neighboring cell volumes :

$$
\widetilde{V}_{i, j, k}=\beta_{i, j, k} V_{i, j, k}+\beta_{i-1, j, k} V_{i-1, j, k}+\beta_{i-1, j-1, k} V_{i-1, j-1, k}+\beta_{i, j-1, k} V_{i, j-1, k},
$$

and the coefficients $\beta_{i, j, k}$ depend on the type of cell $\Omega_{i, j, k}$ :

$$
\beta_{i, j, k}=\left\{\begin{array}{cl}
0 & \text { Solid cells, } \\
1 / 3 & \text { Triangular cells, } \\
1 / 4 & \text { Otherwise, }
\end{array}\right.
$$

These coefficients have been defined in [5] for deriving a conservative discretization of the elastic shear stresses, and then successfully employed in [4] for 2D non-Newtonian computations.

\subsection{Solution strategy and parallel implementation}

The time-advancement of the Navier-Stokes equations is based on the IMEX fractional-step method described in 3 for Newtonian flows and extended in [4] to non-Newtonian flows. For the steady computations reported in the next section, first-order time stepping with Euler schemes is used until the solution reaches steady-state. Unsteady computations of Newtonian flows are performed with second-order methods (BDF2 time-stepping with Adams-Bashforth extrapolations).

The LS-STAG flow solver is a Message Passing Interface (MPI) [17] code written in FORTRAN90 where parallelism is achieved by performing a Cartesian decomposition of the domain. Each cuboid-shaped sub-domain of the decomposition is identified with a coordinate triplet $(I, J, K)$ and is surrounded by one overlapping layer of buffer cells (see Figure 4), which allows to compute the discrete fluxes at the boundary of the sub-domain.

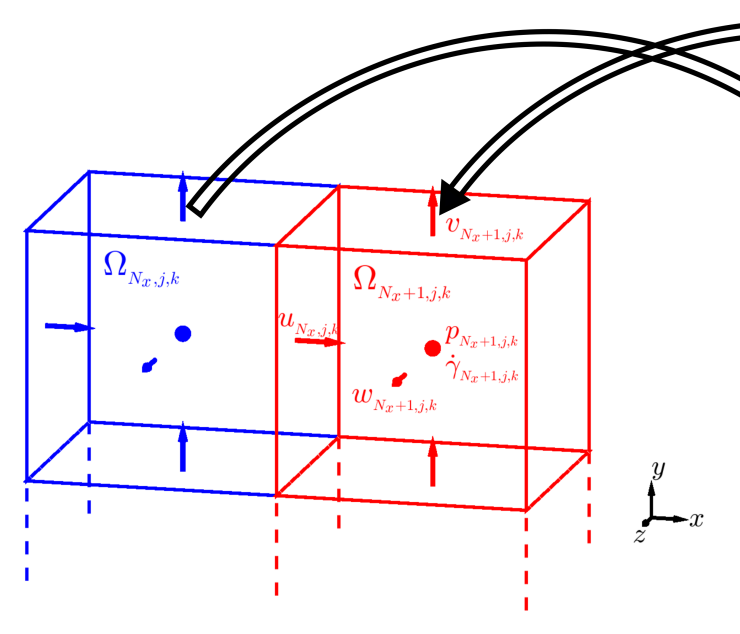

$\operatorname{Sub-domain}(I, J, K)$

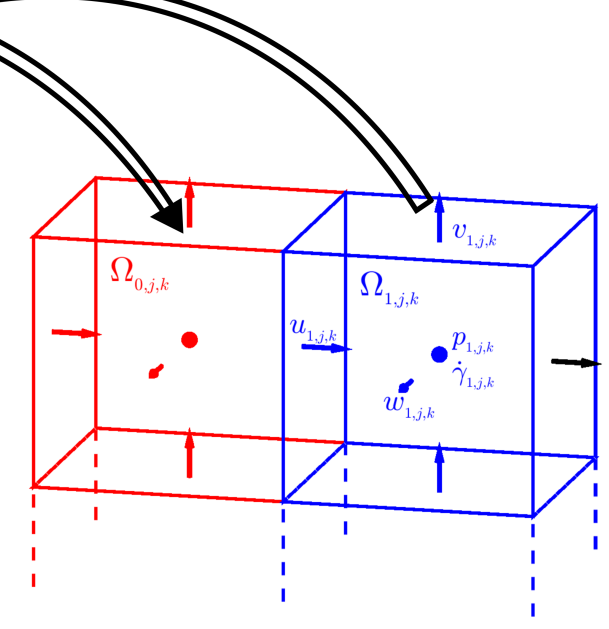

Sub-domain $(I+1, J, K)$

Figure 4: Information interchange between two adjacent sud-domains $(I, J, K)$ and $(I+1, J, K)$. Data in the buffer cells $\Omega_{N_{x}+1, j, k}$ and $\Omega_{0, j, k}$ (in red) are duplicated respectively from the appropriate values in the computational cells $\Omega_{1, j, k}$ and $\Omega_{N_{x}, j, k}$ (in blue). 


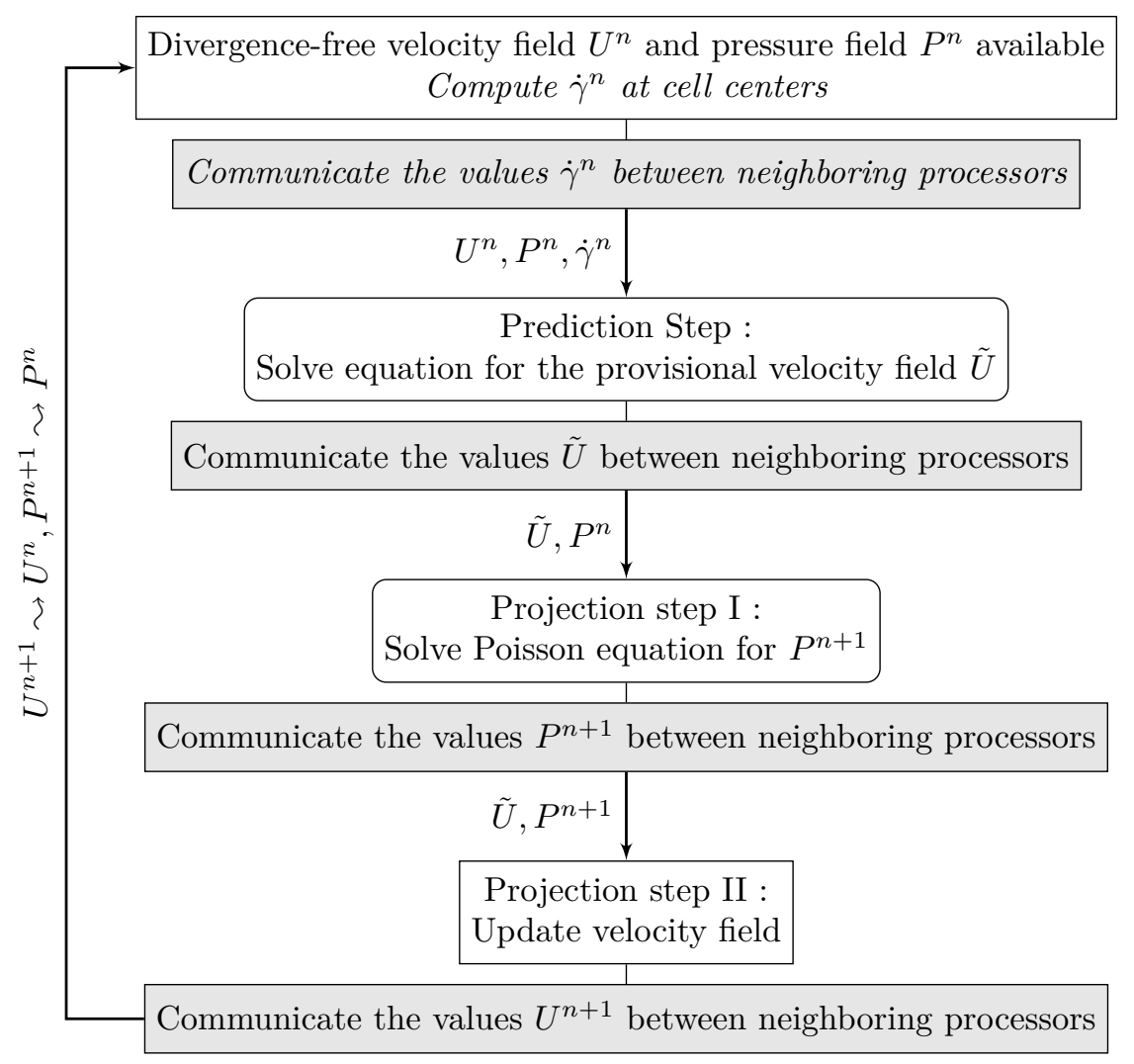

Figure 5: Flowchart of the parallel fractional-step algorithm, the corresponding sequential algorithm is recovered by discarding steps in shaded boxes. The steps marked in italic are required for simulations of non-Newtonian flows only.

The data in the layer of buffer cells are updated from the neighboring sub-domains $(I \pm 1, J \pm$ $1, K \pm 1$ ) by using border exchange communication MPI routines (MPI_SENDRECV) at the end of each key step of the fractional-step algorithm, as summarized in Figure 5 The resulting sequence of operations is the same as for any standard Cartesian method, which notably shows that the LS-STAG cut-cell method introduces no specific issues related to parallelism.

The HYPRE [18] library (V2.10) is employed for the iterative solution of linear systems, most notably the Poisson pressure equation. All the simulations reported in the next section were performed on the computer cluster of Institut Jean Lamour (Université de Lorraine) with an infiniband network and an Intel ${ }^{\circledR}$ Xeon E5-2660 2.20 Ghz processor. A comparison of the LSSTAG solver performance with the commercial CFD code Ansys-FLUENT ${ }^{\circledR}$ will be given in Section 6.2.1

\section{Results and discussion}

The LS-STAG flow solver is now evaluated on various benchmark flows of Newtonian and nonNewtonian fluids in 3D extruded geometries, such as Hagen-Poiseuille flow, flows past a circular cylinder and through a duct with an abrupt expansion. For all computations, the Reynolds number is defined as

$$
R e_{D}=\rho U_{\text {ref }}^{2-n} D^{n} / K
$$

where $D$ and $U_{\text {ref }}$ are reference length and velocity, $n>0$ and $K$ are the power-law parameters (Sect. 2). 


\subsection{Accuracy study of the LS-STAG method}

The spatial accuracy of the LS-STAG method for 2D non-Newtonian fluids has thoroughly been studied on Taylor-Couette flows in [4, where second-order accuracy has been observed in Cartesian cells away from the complex geometry and super-linear convergence in the cut-cells. Although a Taylor-Couette cell is an extruded geometry, the independence of exact solution in the axial direction makes impossible to evaluate the accuracy of LS-STAG discretization in that direction. Hence, a more suitable test-case is the Hagen-Poiseuille flow, where a constant pressure drop drives the flow through a circular cylinder of radius $R$ with no-slip boundaries. For power-law fluids, the analytical solution reads in polar coordinates:

$$
w(r)=w_{\mathrm{c}}\left[1-\left(\frac{2 r}{D}\right)^{\frac{n+1}{n}}\right],
$$

where $w_{\mathrm{c}}$ is the streamwise velocity at the centerline. This test-case has already been considered in [6] for a Newtonian fluid and, following their recommendations, the pipe dimensions are $2 R \times$ $L_{\mathrm{z}}=1 \times 2$ with periodic boundary conditions at the streamwise boundaries. The constant pressure drop is modeled as a source term in the Navier-Stokes equations. Computations are performed at $R e_{D}=2000$, where $D$ and $w_{\mathrm{c}}$ are the reference length and velocity, on a series of refined meshes with square cells of size $h$. In order to evaluate the LS-STAG cut-cell treatment, we have also performed computation with the staircase method which corresponds to a stepwise approximation of the irregular boundaries with Cartesian cells. As a result the staircase treatment only affects the discretization in cut-cells, while it remains unchanged in Cartesian cells.

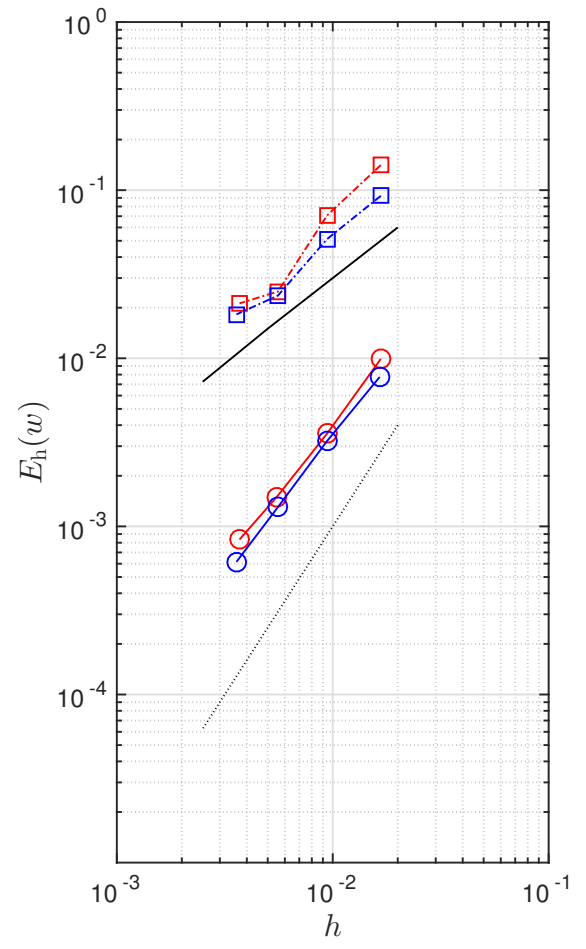

(a)

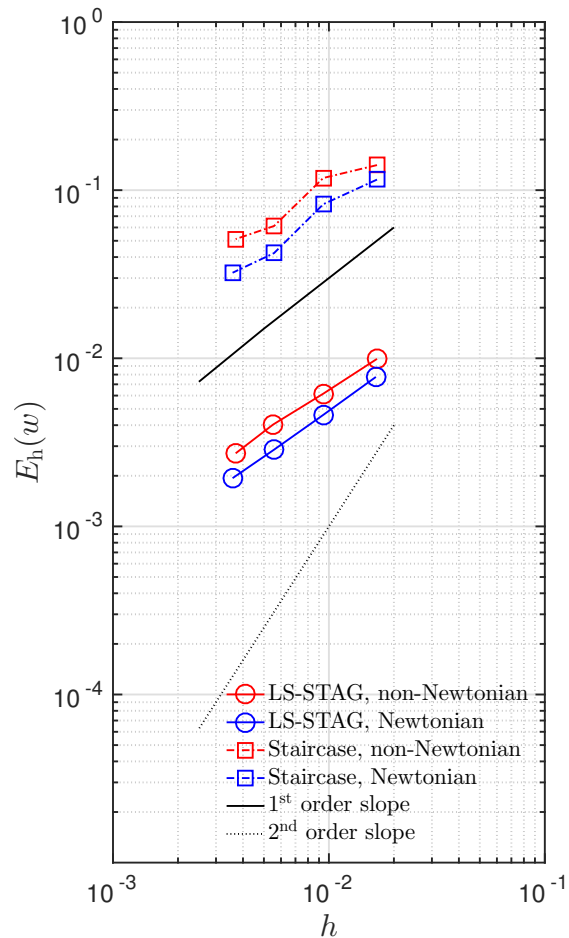

(b)

Figure 6: Maximal error on the streamwise velocity versus grid size $h$ for the Hagen-Poiseuille flow. At left: on $90 \%$ of the fluid domain; at right: on the whole fluid domain.

Figure 6 compares the errors on the streamwise velocity measured in the maximum norm on the whole computational domain and in Cartesian cells away from the irregular boundary for 
both Newtonian $(n=1)$ and shear-thinning $(n=0.5)$ fluids. As in the 2D case [4], the LSSTAG errors are more than a decade lower than those of the first-order staircase method, which highlights the benefits of our cut-cell treatment. Second order accuracy is obtained in Cartesian cells away from the irregular boundary for both fluid types. The magnitude of errors are slightly higher for the power-law fluids due to additional errors introduced by the computation of the nonNewtonian viscosity. However, compared to the 2D case, the error on the whole domain (which actually measures the error in cut-cells) reduces from superlinear to linear only. This behavior, also observed in the cut-cell method of Ref. [6], is certainly caused by the ad hoc computation of the off-diagonal gradients $\partial w / \partial x$ and $\partial w / \partial y$ (see Sect. 5.2 which were absent from the $2 \mathrm{D}$ version.

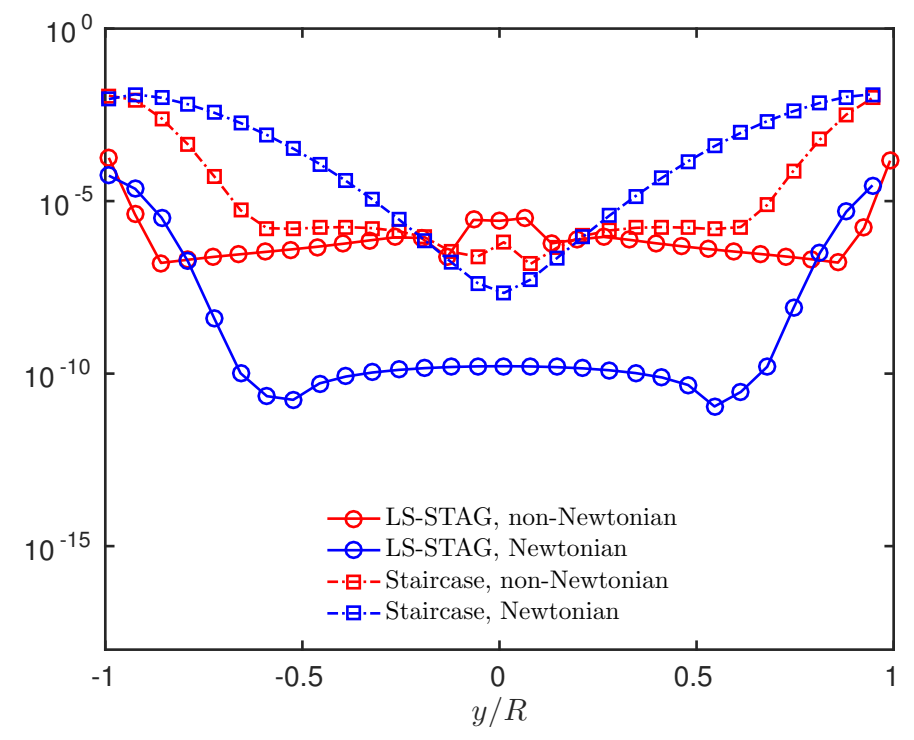

Figure 7: Pointwise error profile for $h=5.5 \times 10^{-3}$ on cross-section $z=1$ of Hagen-Poiseuille flow.

Further insight on the structure of discretization error is given in Figure 7, which displays the pointwise error on mesh points along cross-section $z=1$ for $h=5.5 \times 10^{-3}$. First, it can be observed that the largest errors are localized near the complex boundary and, for the LS-STAG method, the errors drop to several orders of magnitude away from the cut-cells. In contrast, the crude treatment of the staircase method pollutes the solution in the whole fluid domain. For nonNewtonian fluids, the difference between the staircase and LS-STAG method is less pronounced. This trend was also observed in the $2 \mathrm{D}$ version [4, and is attributed to the additional errors introduced by the evaluation of the non-Newtonian viscosity in the viscous fluxes.

\subsection{Flows past circular cylinder}

In this part, we consider the flow past circular cylinder at two different flow regimes: an unsteady laminar flow with time-varying Reynolds number and a weakly turbulent flow. The computational domain is depicted in Figure 8; its dimensions and conditions at the boundaries will be given below for each configuration.

\subsubsection{Comparison with unstructured body-fitted solver Ansys-FLUENT우}

The purpose of this part is to address 2 crucial questions regarding flow computations in complex geometries which, to the best of our knowledge, have seldomly been addressed in the literature: how accurate is the solution given by a cut-cell/IBM code compared to a body-fitted unstructured solver of same formal order of accuracy? How do the computational resources of the cut-cell/IBM code (memory usage, CPU time) compare with those of the body-fitted solver?

For that purpose, we have conducted a series of computations with the LS-STAG code and the commercial CFD package FLUENT ${ }^{\circledR}$ (V14.57) on the benchmark 3D-3Z initially proposed in 


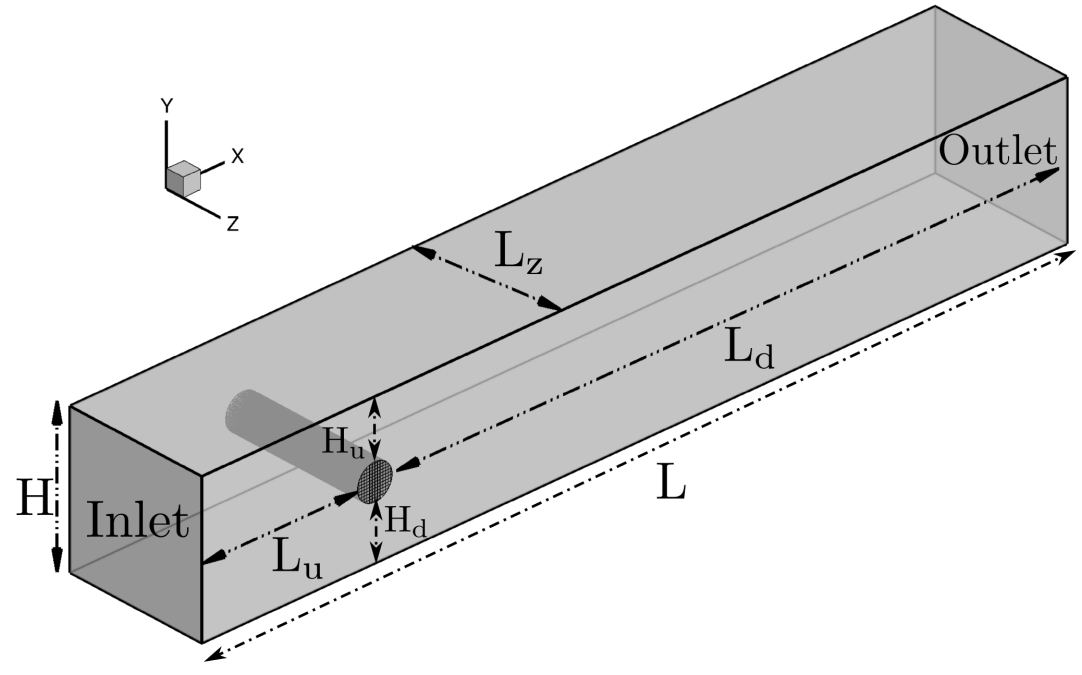

Figure 8: Computational domain for the cylinder flows of Section 6.2

[19, which concerns the unsteady laminar flow past a circular cylinder in a confined square duct. The computational domain is depicted in Figure 8, with spanwise and cross-stream dimensions $H=0.41 \mathrm{~m}$ and $L_{\mathrm{z}}=0.41 \mathrm{~m}$, respectively. The upstream and downstream distances of the cylinder (of diameter $D=0.1 \mathrm{~m}$ ) are set to $L_{\mathrm{u}}=0.45 \mathrm{~m}$ and $L_{z}=1.95 \mathrm{~m}$ with an $1.0 \mathrm{~cm}$ offset in the cross-stream direction $\left(H_{\mathrm{d}}=0.15 \mathrm{~m}\right.$ and $\left.H_{\mathrm{u}}=0.16 \mathrm{~m}\right)$. At the spanwise and cross-stream boundaries, no-slip conditions are applied, while a parabolic stream-wise velocity profile is set at the inlet:

$$
u_{\text {inlet }}=16 U_{\max } y z \sin \left(\frac{\pi t}{8}\right) \frac{(H-y)(H-z)}{H^{4}}, \quad U_{\max }=2.25 \mathrm{~m} / \mathrm{s} .
$$

At the outlet, a convective outflow condition [20] is applied. The fluid viscosity is $\nu=10^{-3} \mathrm{~m}^{2} / \mathrm{s}$, yielding a time-varying Reynolds number $\operatorname{Re}(t) \in[0,100]$ based on the cylinder diameter $D$ and the mean flow velocity $U_{\text {ref }}=4 U_{\max } / 9$ at the inlet.

For this configuration, Bayraktaret al. [7] recently conducted a thorough comparison of the performances of 3 unstructured CFD packages (OpenFOAM, Ansys-CFX and FEATFlow) on a sequence of body-fitted curvilinear grids and provided highly accurate benchmark results. The same grids have been used for our FLUENT ${ }^{\circledR}$ computations: a $x y$ view of the coarsest mesh we considered, labeled L2 in [7, is shown in Figure 9(a) The refined grid layer around the cylinder surface consists in near-uniform hexahedral cells of size $\Delta_{2} / D \simeq 0.31$ (based on the main diagonal) at the cylinder surface. The 3D mesh is obtained by extrusion in the $z$ direction with a uniform distribution of cells, which gives a number of 49152 3D cells. Finer meshes of cell size $\Delta_{2} / 2$ and $\Delta_{2} / 4$ are obtained by successive refinement, yielding meshes L3 and L4 with 393216 and 3145728 cells respectively. Corresponding block-structured Cartesian grids M2-M4 have been constructed for the LS-STAG solver with the following constraints: the total number of fluid cells and the grid resolution near the complex boundary should be as close as possible to the curvilinear meshes L2-L4, in spite of the inferior flexibility of Cartesian grids. The $x y$ view of grid M2 is shown in Figure 9(b) : grid clustering around the cylinder is obtained by defining a rectangular block of $L_{x} / D \times L_{y} / D=4.0 \times 2.0$ with uniform cells of size $h_{x} / D \times h_{y} / D=0.118 \times 0.105$, while the grid size is relaxed upstream and downstream of this block. In the spanwise direction, a uniform cell distribution of size $\Delta z / D=0.256$ is used. The resulting 3D mesh has 49136 fluid cells of average size $\Delta_{2} / D \simeq 0.30$ near the cylinder body, which is similar to the L2 mesh. The main characteristics of the M2-M4 meshes are given in Table 1 along with the time-step used for the LS-STAG and FLUENT ${ }^{\circledR}$ computations.

The FLUENT $^{\circledR}$ computations have been performed with the pressure-based solver, with 

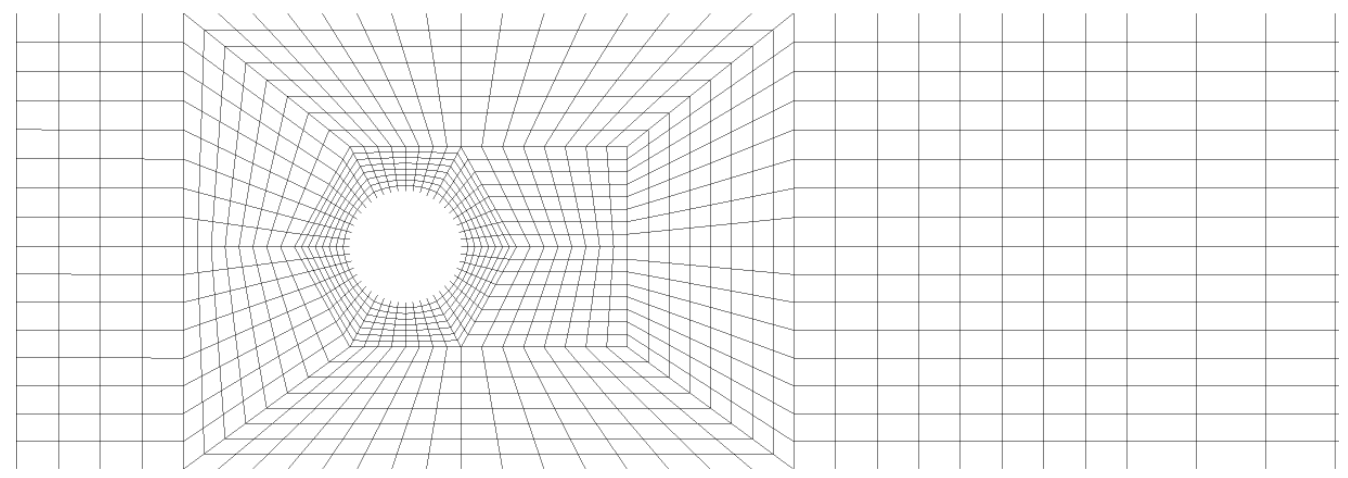

(a)

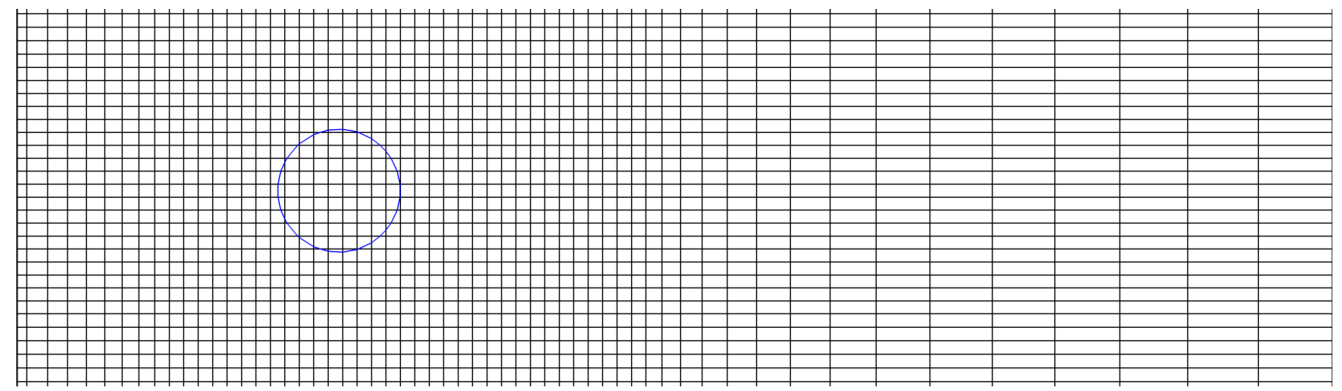

(b)

Figure 9: 2D view of the coarsest meshes for the circular cylinder benchmark: (a) FLUENT ${ }^{\circledR}$ L2 mesh and (b) LS-STAG M2 mesh.

Table 1: Salient properties of the LS-STAG meshes used for the circular cylinder benchmark.

\begin{tabular}{|c|c|c|c|c|c|c|c|}
\hline \multirow{2}{*}{ Mesh } & \multirow{2}{*}{$\Delta t U_{\text {ref }} / D$} & \multirow{2}{*}{$N_{x} \times N_{y} \times N_{z}$} & \multicolumn{3}{|c|}{ Cell Types } & \multirow{2}{*}{ \# fluid cells } & $h_{x} / D \times h_{y} / D$ \\
\cline { 4 - 5 } & & & Cartesian & Cut-cell & Solid & & \\
\hline M2 & 0.016 & $83 \times 38 \times 16$ & $97.4 \%$ & $1.1 \%$ & $1.5 \%$ & 49136 & $0.118 \times 0.105$ \\
\hline M3 & 0.008 & $159 \times 77 \times 32$ & $97.6 \%$ & $0.6 \%$ & $1.8 \%$ & 391776 & $0.060 \times 0.051$ \\
\hline M4 & 0.004 & $317 \times 153 \times 64$ & $97.8 \%$ & $0.3 \%$ & $1.9 \%$ & 3104064 & $0.030 \times 0.026$ \\
\hline
\end{tabular}

second-order spatial discretizations [21]: QUICK scheme for convection discretization, GreenGauss node-based interpolation for gradient calculation, and PRESTO! for pressure interpolation. Second-order implicit time-stepping (BDF2) has been selected. For pressure-velocity coupling, 2 variants of the segregated PISO time-advancement have been evaluated: the iterative (default) and non-iterative (NITA) versions. The default PISO algorithm performs several inner iterations per time-step to solve the momentum and pressure equations while the NITA variant solves these equations only once per time-step, yielding thus a time-advancement which resembles the most to our AB/BDF2 fractional step method. All other numerical parameters have been left to their default settings.

As in [7, 19, the LS-STAG and FLUENT ${ }^{\circledR}$ simulations are conducted for the time interval $[0,8 \mathrm{~s}]$ corresponding to a half period of the inflow condition $(35)$, and are evaluated against a highly accurate FEATFLOW solution obtained on a fine L5 mesh [22]. Figure 10 displays the time evolution of the force coefficients obtained on the various meshes. First, it can be seen that both LS-STAG and FLUENT ${ }^{(C)}$ solvers fail to capture the flow dynamics on the M2/L2 mesh due to insufficient grid resolution. Accurate results are obtained on the 2 finer meshes although, as shown in the close-ups of Figure 10, the LS-STAG results appear to be slightly inferior to FLUENT ${ }^{\complement}$. A quantitative comparison is provided by Table 2 where, as proposed in Ref. [7], 

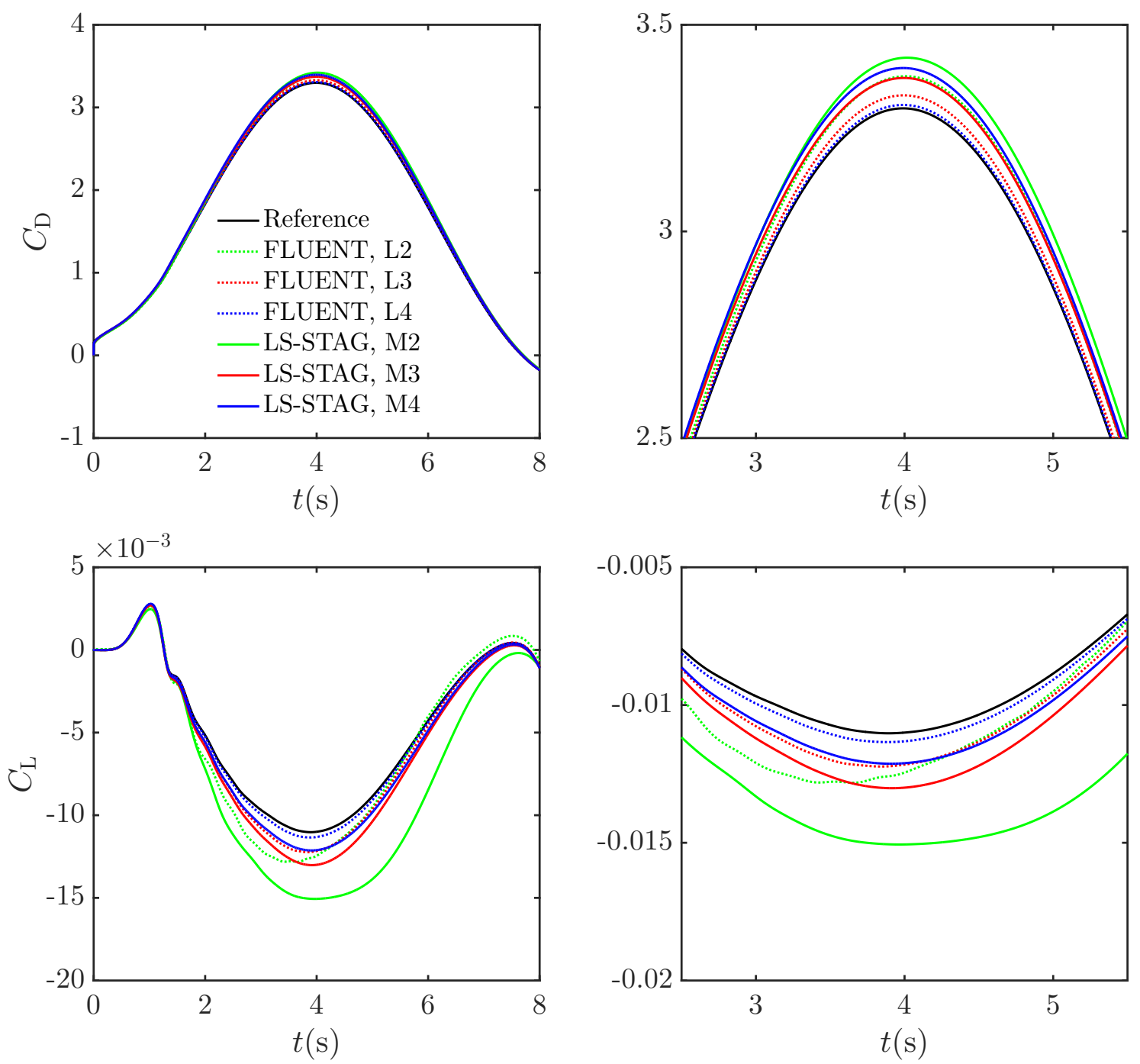

Figure 10: Time evolution of the force coefficients computed by LS-STAG, FLUENT ${ }^{\circledR}$, and reference solution 22 at the cylinder. At left: on the total time interval; at right: close up around $t=4 \mathrm{~s}$.

Table 2: Relative errors (in percent) of the force coefficients with respect to the reference solution $[22]\left(C_{D_{\max }}=\right.$ $3.2978, C_{L_{\max }}=0.00276$ and $\left.C_{L_{\min }}=-0.0110\right)$ and $L_{2}$ errors for $t \in[0,8 \mathrm{~s}]$.

\begin{tabular}{|c|c|c|c|c|c|c|}
\hline \multirow{2}{*}{ Solver } & \multirow{2}{*}{ Mesh } & \multicolumn{3}{|c|}{ \% Error } & $\% L_{2}$ & Error \\
\cline { 3 - 7 } & & $C_{\mathrm{D}_{\max }}$ & $C_{\mathrm{L}_{\max }}$ & $C_{\mathrm{L}_{\min }}$ & $C_{\mathrm{D}}$ & $C_{\mathrm{L}}$ \\
\hline \multirow{3}{*}{ LS-STAG } & $\mathrm{M} 2$ & 3.72 & 11.05 & 36.61 & 3.74 & 48.06 \\
\cline { 2 - 7 } & $\mathrm{M} 3$ & 2.23 & 2.96 & 18.07 & 2.42 & 16.94 \\
\cline { 2 - 7 } & $\mathrm{M} 4$ & 2.96 & 0.49 & 10.02 & 3.05 & 10.22 \\
\hline \multirow{3}{*}{ FLUENT $^{\odot}$} & $\mathrm{L} 2$ & 2.35 & 4.40 & 16.29 & 2.11 & 17.03 \\
\cline { 2 - 7 } & $\mathrm{L} 3$ & 0.96 & 2.16 & 10.96 & 0.81 & 10.01 \\
\cline { 2 - 7 } & $\mathrm{L} 4$ & 0.25 & 0.08 & 2.89 & 0.21 & 2.67 \\
\hline
\end{tabular}

errors on the extrema of force coefficients and $L_{2}$ errors for $t \in[0,8 \mathrm{~s}]$ are compared. The LSSTAG results display a monotonic convergence for the lift coefficient, $C_{\mathrm{L}_{\min }}, C_{\mathrm{L}_{\max }}$ and $C_{\mathrm{L}}$. The errors are comparable to FLUENT ${ }^{\circledR}$ on the first 2 meshes, and only slightly inferior to FLUENT ${ }^{\circledR}$ on M4/L4. In addition, results on the drag coefficient, $C_{D_{\max }}$ and $C_{\mathrm{D}}$, although satisfactory, do 
not display a grid convergence as smooth as FLUENT ${ }^{\odot}$. As already observed in Sect. 6.1. this behavior is certainly due to the ad hoc discretization of the off-diagonal gradients $\partial w / \partial x$ and $\partial w / \partial y$ in the cut-cells.

Finally, Table 3 presents the run-time and memory usage for the LS-STAG and FLUENT ${ }^{(}$ computations. Due to the limited availability of FLUENT ${ }^{\circledR}$ licenses in our institution, the comparisons have been performed on 2 processors for the coarse mesh computation (M2 and L2) and 4 processors for the two finer meshes. The FLUENT ${ }^{\circledR}$ computations with the default PISO algorithm proved to be exceedingly time consuming (more than 22 times slower than LS-STAG) due to the cost of inner iterations and have not been pursued on the finest mesh. Compared to the NITA variant of the PISO algorithm, the LS-STAG code is still substantially faster (approximately 3 to 4 times faster than FLUENT $\left.{ }^{(}\right)$). The memory savings of the LS-STAG methods are also sub-

Table 3: Clock time and memory usage of the LS-STAG and FLUENT ${ }^{\odot}$ computations on the IJL-LEMTA parallel machine ( $n p$ refers to the number of processors).

\begin{tabular}{|c|c|c|c|c|c|c|c|}
\hline \multirow{2}{*}{ Mesh } & \multirow{2}{*}{$n p$} & \multicolumn{3}{|c|}{ Wall Time (min) } & \multicolumn{3}{|c|}{ Memory Usage (mb) } \\
\cline { 4 - 6 } & \multirow{2}{*}{} & \multirow{2}{*}{ LS-STAG } & \multicolumn{2}{|c|}{ FLUENTC } & \multirow{2}{*}{ LS-STAG } & \multicolumn{2}{|c|}{ FLUENTC } \\
\cline { 4 - 5 } & & & PISO & NITA & & PISO & NITA \\
\hline M2/L2 & 2 & 16 & 363 & 55 & 90 & 406 & 400 \\
\hline M3/L3 & 4 & 132 & 2943 & 488 & 411 & 1368 & 1388 \\
\hline M4/L4 & 4 & 2164 & - & 7841 & 2417 & - & 7927 \\
\hline
\end{tabular}

stantial (4.5 times less memory needed than FLUENT ${ }^{\circledR}$ on $\mathrm{M} 2 / \mathrm{L} 2$ and superior to 3 times for the finer meshes) due to the on-the-fly computation of cell geometry parameters of the LS-STAG code. Finally, we mention that these comparisons are made with the current implementation of LS-STAG code that uses the HYPRE library for solving the linear systems. Since these linear systems typically take up to $60 \%$ of the computational cost of a simulation, the optimization of LS-STAG code will always be limited by the performances of its algebraic solver package.

\subsubsection{Turbulent flow at $\operatorname{Re}_{D}=300$}

The purpose of this section is to assess the ability of LS-STAG method to perform accurate direct numerical simulation (DNS) of turbulent flows in complex geometries. The selected testcase is the (weakly) turbulent flow at $\mathrm{Re}=300$, at beginning of the wake turbulent shedding regime [23], for which accurate treatment of the fine scale structures in the near-cylinder wake is needed to properly capture the flow dynamics. It has been selected due to the availability of experimental results 24] and accurate body-conforming DNS (the spectral simulations of Mittal \& Balachandar [8] and the zonal B-splines computations of Kravchenko et al. [9]). These studies will serve as reference for our simulations. In addition, this flow has also been computed by a state-of-the-art immersed boundary method [10. In order to address the comparison of LS-STAG method with this IBM computation, we have used the same domain definition $(L=50 D, H=30 D$ and $L_{\mathrm{z}}=6 D$ in Figure 8D and built Cartesian grids with similar resolution. The coarse mesh M1 (correspond to the Case 1 mesh in [10]) has $243 \times 133 \times 64$ cells and a grid distribution similar to the mesh displayed in Figure 9(b) the grid distribution is uniform in the spanwise direction, and in the $x y$ plane a uniform block of size $4 D \times 4 D$ is used around the circular cylinder with grid resolution $h / D=3.88 \times 10^{-2}$. A finer mesh M2 of $422 \times 226 \times 64$ cells with grid resolution $h / D=1.94 \times 10^{-2}$ near the cylinder (similar to Case 2 in [10]) has also been considered.

The boundary conditions are the following: uniform velocity $u=U_{\text {ref }}$ at the inlet boundary, periodicity at the spanwise boundaries, traction-free conditions at cross-stream boundaries, and convective outflow condition at the outlet boundary. The time step is set as $\Delta t U_{\text {ref }} / D=9 \times 10^{-3}$ and $4.5 \times 10^{-3}$ on mesh M1 and M2 respectively, yielding a maximal cell CFL number of 0.41 during one shedding period. The initial condition is defined as the rest with random perturbations in the spanwise and crosswise direction to trigger the flow instability. The simulations were advanced in time up to a statistically steady flow pattern. Then, statistics were calculated by averaging in 


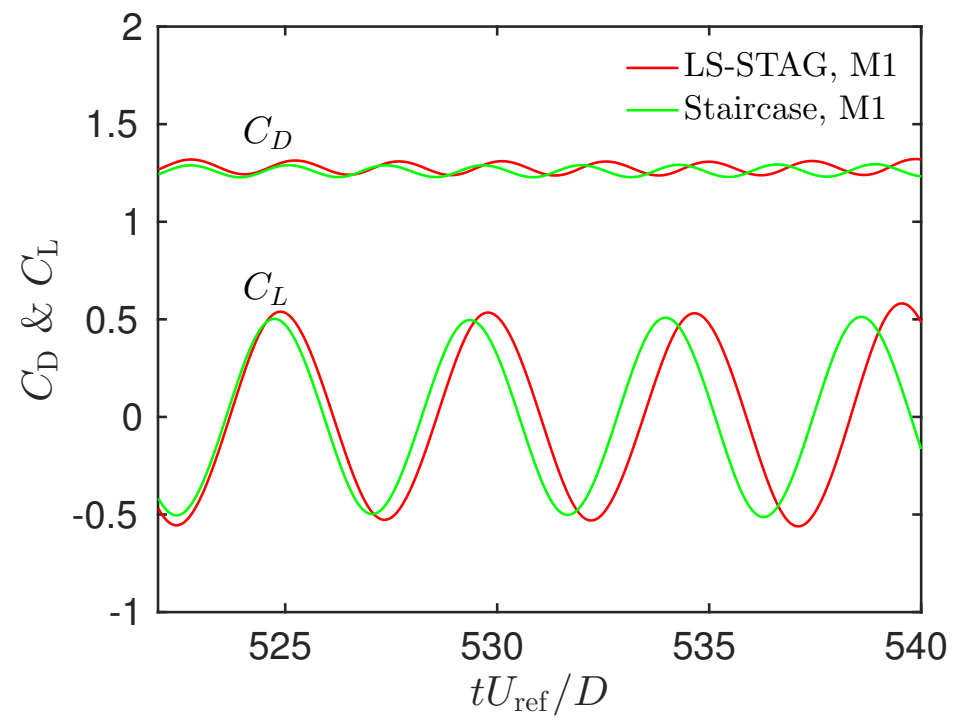

Figure 11: Time evolution of the force coefficients for the cylinder flow at $R e_{D}=300$ on the M1 mesh.

space in the spanwise direction and in time over 15 shedding cycles $\left(T \sim 80 D / U_{\text {ref }}\right)$. In addition to LS-STAG computations on M1 and M2 meshes, a staircase simulation (see Section 6.1) on M1 mesh has also been performed in order to evaluate the benefits of our cut-cell treatment. For both methods, the time history of the drag and lift coefficients is displayed in Figure 11] Although both methods give force signals with comparable amplitudes, the crude staircase treatment of the complex geometry introduces a spurious lag in the force signals which yield incorrect prediction of the shedding frequency, as we shall see next.

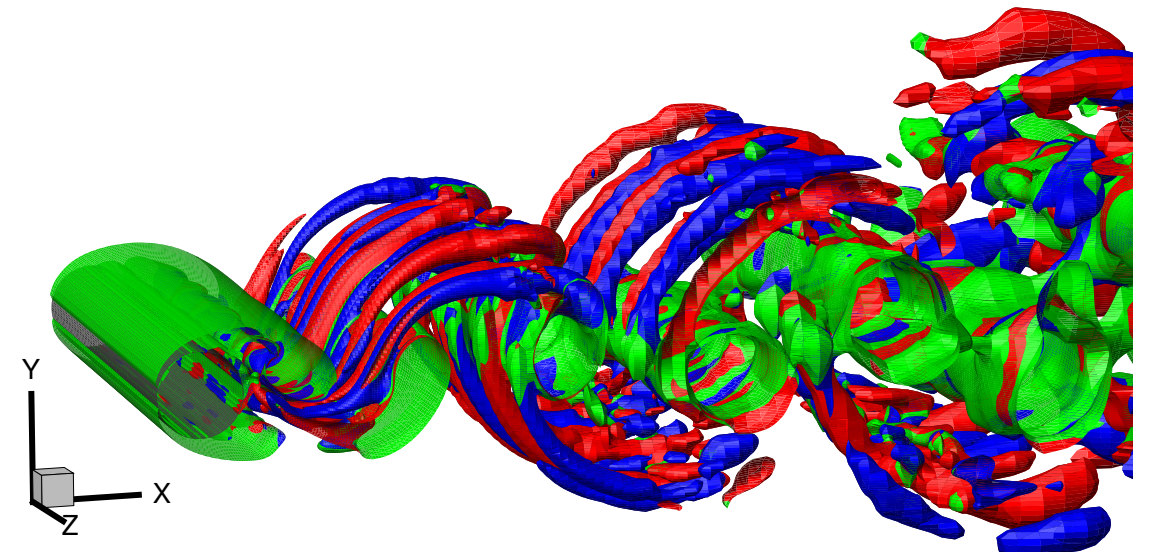

Figure 12: Vortex visualization of the cylinder flow at $R e_{D}=300$ by the $Q$-criterion on mesh M2. As in [10], the isosurfaces displayed correspond to the value $Q=0.1$ and are colored with streamwise vorticity values: red is $\omega_{x} D / U_{\text {ref }}=0.25$ and blue is $\omega_{x} D / U_{\text {ref }}=-0.25$.

The flow at $R e_{D}=300$ is characterized by the presence of streamwise-oriented coherent structures of spanwise length order of $D[23$ in the cylinder wake. These large vortical structures are best visualized by the $Q$-criterion [25, which identifies vortical flow regions to positive values of the second invariant of the velocity gradient $Q$. Figure 12 displays isosurfaces of $Q$ in the near-wake of circular cylinder at an instantaneous time. It clearly shows the presence of large counter-rotating streamwise vortices in the near cylinder wake, whose number decreases further downstream. A similar qualitative observation of the wake structure has been performed in 9 by visualizing the 
instantaneous streamwise vorticity.

Table 4: Mean flow parameters for the cylinder flow at $R e_{D}=300$

\begin{tabular}{|c|c|c|c|}
\hline Case & $\bar{C}_{\mathrm{D}}$ & $\bar{C}_{\mathrm{L}_{\mathrm{rms}}}$ & $S t$ \\
\hline Measurement [26] & 1.22 & - & - \\
\hline Measurement [24] & - & 0.435 & 0.203 \\
\hline Spectral simulation [8] & 1.26 & 0.380 & 0.203 \\
\hline B-spline simulation [9] & 1.28 & 0.40 & 0.203 \\
\hline LS-STAG, M1 & 1.29 & 0.420 & 0.203 \\
\hline LS-STAG, M2 & 1.29 & 0.437 & 0.203 \\
\hline Staircase, M1 & 1.24 & 0.346 & 0.217 \\
\hline IBM Case 1 [10] & 1.41 & 0.44 & 0.20 \\
\hline IBM Case 2 [10] & 1.27 & 0.42 & 0.21 \\
\hline Body-fitted finite-volume [27] & 1.28 & 0.499 & 0.195 \\
\hline
\end{tabular}

Table 4 compares the main flow characteristics, namely the mean drag coefficient, $\bar{C}_{\mathrm{D}}$, root mean square average of the lift coefficient, $\bar{C}_{\mathrm{L}_{\mathrm{rms}}}$, and the Strouhal number $S t=f D / U_{\text {ref }}$, where $f$ is the shedding frequency, with reference body-fitted simulations [8, 9, experimental measurements 26, 24, IBM computations [10] and the recent DNS of Rajani et al. 27] with a finite-volume solver on multiblock polar grids. It can be clearly seen that the shedding frequency is the easiest quantity to predict: only the IBM and staircase method on the coarsest mesh M1 and Ref. [27] misestimate its value. For the drag coefficient, accurate reference computations bracket its value in the range $[1.26,1.28]$, for which the LS-STAG computations on both coarse and fine meshes give a very close prediction, $\bar{C}_{\mathrm{D}}=1.29$. The $\mathrm{rms}$ lift coefficient proves to be the most sensitive value to predict, with reference values spread in the range [0.380,0.435], with an upper value given by the experiments [24]. Both coarse and fine mesh LS-STAG simulation fall in this range, and in particular the M2 prediction is the closest computational result to the experimental value.
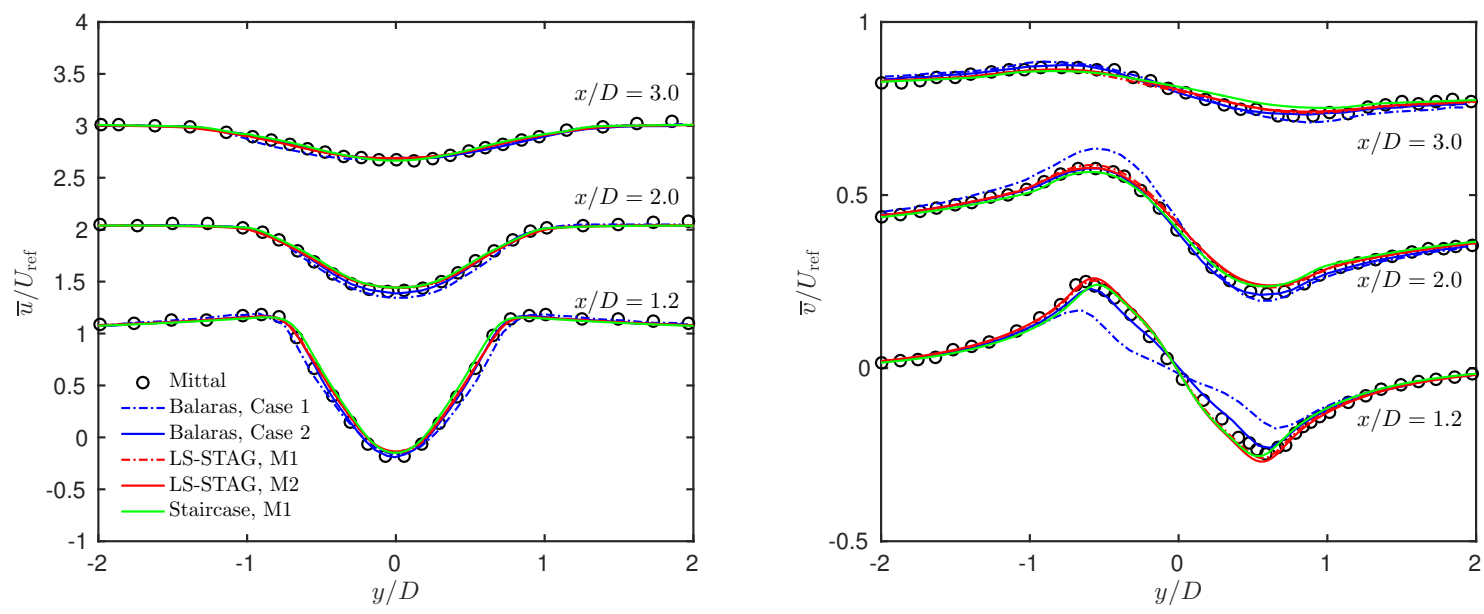

Figure 13: Mean velocity profiles at different locations in the wake of circular cylinder at $R e_{D}=300:$ stream-wise (left) and cross-flow (right) components.

The mean velocity profiles, time-averaged velocity fluctuations and Reynolds shear stress at three different downstream locations in the wake of circular cylinder are displayed in Figures 13 to 15 respectively. The LS-STAG and staircase computations are compared to IBM simulations [10] and reference spectral results [8] (The B-spline solution of Ref. [9] are virtually indistinguishable from the spectral data and not shown in these figures). Firstly, it can be observed that the LS-STAG results on both coarse and fine meshes show excellent agreement with the reference 

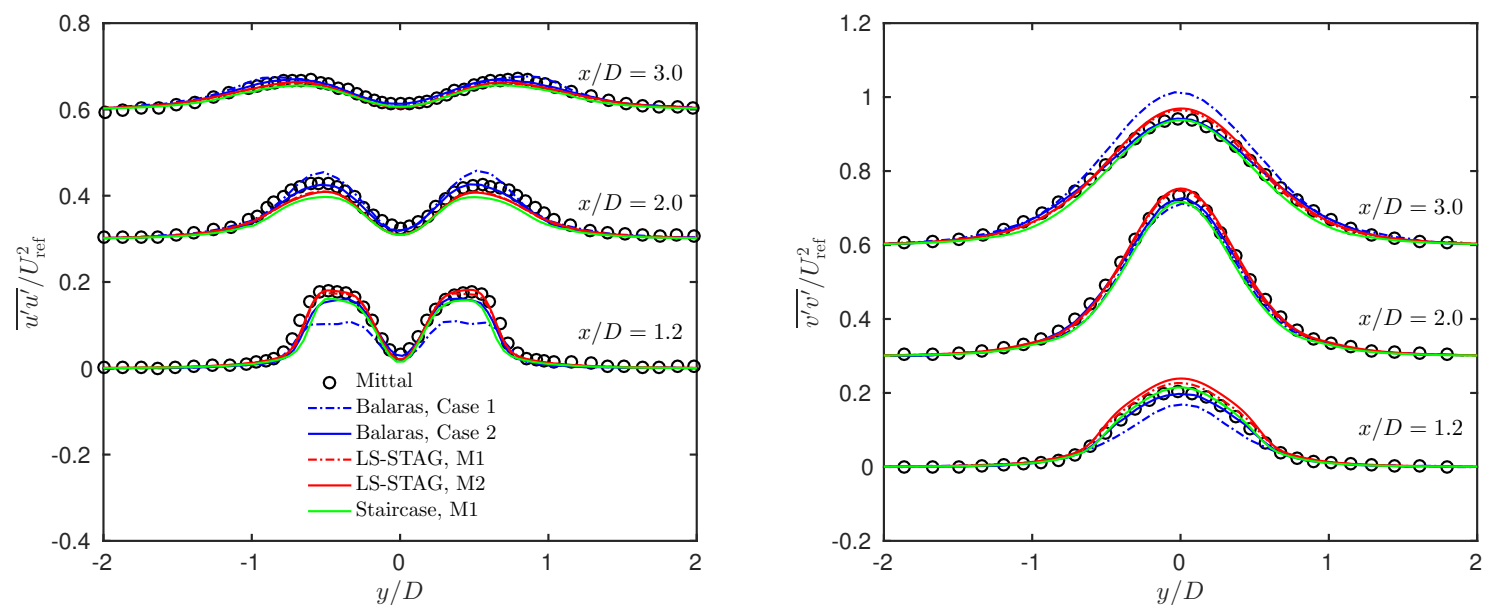

Figure 14: time-averaged velocity fluctuations at different locations in the wake of circular cylinder at $\operatorname{Re}_{D}=300$. At left: stream-wise component and right: cross-flow component.

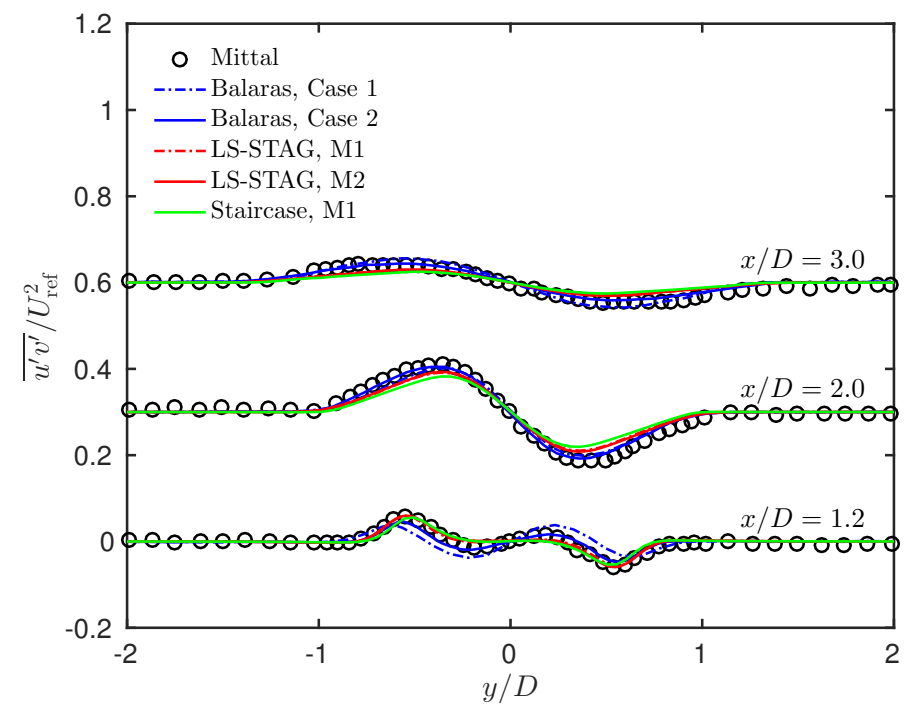

Figure 15: time-averaged Reynolds shear stress at different locations in the wake of circular cylinder at $\operatorname{Re}_{D}=300$.

results, which indicates that the M1 grid resolution is sufficiently fine to capture the wake dynamics at this flow regime. For this grid resolution, the IBM computation exhibits notable discrepancies on the cross-flow velocities (Figure 13 ) and velocity fluctuations (Figure 14) in the cylinder wake. It was conjectured that these discrepancies were caused by insufficient grid resolution, see [10]. However, on the light of LS-STAG computations, these discrepancies are more likely due to the inferior treatment of complex geometries given by the IBM compared to a cut-cell method of formally same order of accuracy. It is also interesting to observe that the staircase method, which is formally first-order accurate according to Section 6.1 gives superior results to the IBM method at similar grid resolution, see Table 4 and Figures 13 and 14

\subsection{Flow of shear-thinning liquids in a 1:2 axisymmetric sudden expansion}

Finally, the LS-STAG method is applied to the flow of Newtonian and shear-thinning fluids in an axisymmetric sudden expansion geometry of ratio $\mathrm{ER}=2$, for which rheological characterization, experimental flow measurements (nuclear magnetic resonance, NMR) and FLUENT ${ }^{\circledR}$ simulations have recently been performed by Rigal [11. Following this work, the geometry 

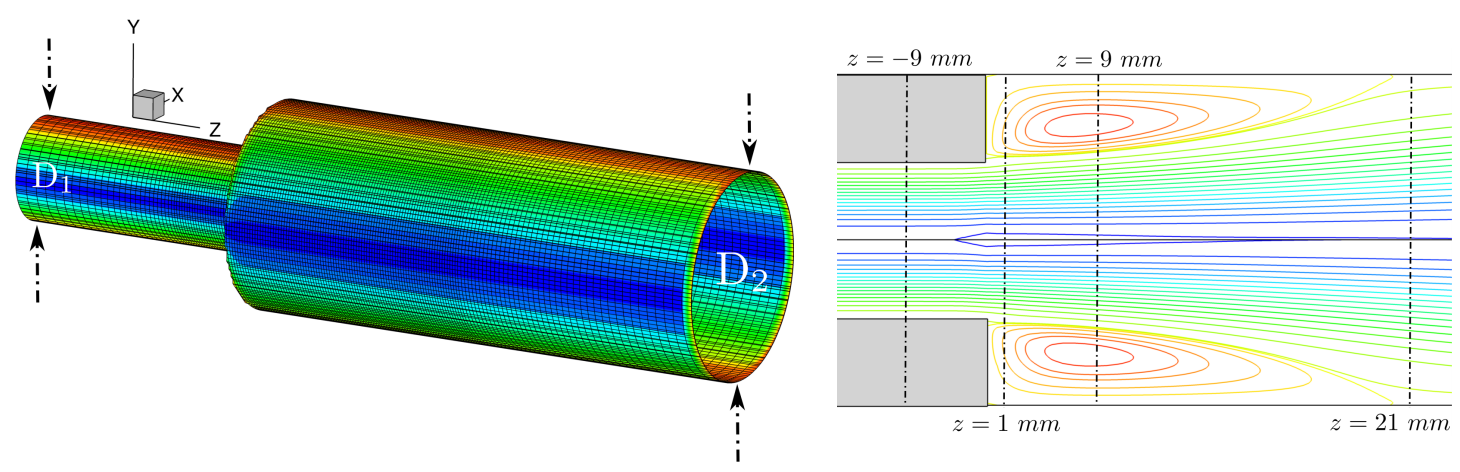

Figure 16: Sketch of the axisymmetric sudden expansion geometry: At left: isometric view and at right: 2D view. The sudden expansion plane is placed at $z=0$.

sketched in Figure 16 is composed by two pipes of diameter $D_{1}=D_{2} / 2=10 \mathrm{~mm}$, with upstream $\left(L_{\mathrm{u}}=150 \mathrm{~mm}\right)$ and downstream $\left(L_{\mathrm{d}}=300 \mathrm{~mm}\right)$ lengths set sufficiently large to allow complete flow development. At the pipe inlet, an Ostwald velocity profile is imposed:

$$
w(r)=\frac{3 n+1}{n+1} U_{\text {ref }}\left[1-\left(\frac{2 r}{D_{1}}\right)^{\frac{n+1}{n}}\right],
$$

where $U_{\text {ref }}$ is the inlet mean velocity and $n$ is the power-law index. 3 types of fluids are considered: water (Newtonian) and two Xanthan solutions (of $0.1 \%$ and $0.3 \%$ mass concentration) whose shearthinning behavior has been fitted with the power-law (4) and Cross (5) models in Table 5 The power-law parameters are used to calculate the flow Reynolds number 33 and inlet velocity 36 . while the Cross model is used to model the shear-thinning viscosity in the LS-STAG computations.

Table 5: Parameters of the power-law and Cross model reported by Rigal [11] for the 2 Xanthan solutions.

\begin{tabular}{|c|c|c|c|c|c|c|}
\hline Fluid type & $n$ & $k\left(\right.$ Pa.s $\left.^{n}\right)$ & $\eta_{0}($ Pa.s $)$ & $\eta_{\infty}($ Pa.s $)$ & $m$ & $K(\mathrm{~s})$ \\
\hline Xanthane $0.1 \%$ & 0.543 & 0.128 & 0.376 & 0.00193 & 0.631 & 1.64 \\
\hline Xanthane $0.3 \%$ & 0.322 & 1.11 & 9.84 & 0.0048 & 0.764 & 13.1 \\
\hline
\end{tabular}

First, a grid convergence study is conducted on 4 refined meshes whose properties are reported in Table 6 In the axial direction, the grid size $h_{z}$ is kept constant in the vicinity of the singular expansion $(z \in[-5 \mathrm{~mm}, 15 \mathrm{~mm}])$, and is relaxed upstream and downstream. In the other 2 directions, the grid is uniform of size $h$.

Table 6: Main properties of the grids for axisymmetric expansion flow.

\begin{tabular}{|c|c|c|c|c|c|c|}
\hline \multirow{2}{*}{ Mesh } & \multirow{2}{*}{$N_{x} \times N_{y} \times N_{z}$} & \multicolumn{3}{|c|}{ Cell Types } & \multirow{2}{*}{$D_{1}$} & \multirow{2}{*}{$D_{z} / D_{1}$} \\
\cline { 3 - 5 } & & Cartesian & Cut-cell & Solid & & \\
\hline M1 & $50 \times 50 \times 185$ & 254844 & 32336 & 175320 & $4.08 \times 10^{-2}$ & $16.7 \times 10^{-2}$ \\
\hline M2 & $100 \times 100 \times 370$ & 2147480 & 130200 & 1422320 & $2.02 \times 10^{-2}$ & $8.33 \times 10^{-2}$ \\
\hline M3 & $150 \times 150 \times 555$ & 7321320 & 294780 & 4871400 & $1.35 \times 10^{-2}$ & $5.56 \times 10^{-2}$ \\
\hline M4 & $200 \times 200 \times 740$ & 17428564 & 523916 & 11647520 & $1.01 \times 10^{-2}$ & $4.17 \times 10^{-2}$ \\
\hline
\end{tabular}

For the Xanthan $0.1 \%$ flow at $R e_{D_{1}}=56$, Figure 17 displays axial velocity profiles at various cross sections (defined in Figure 16(right)) given by the LS-STAG computations and the experiments. Negative velocities at $z=1 \mathrm{~mm}$ and $z=9 \mathrm{~mm}$ indicate the presence of a recirculation zone which vanishes downstream $(z=21 \mathrm{~mm})$. An excellent agreement is observed with the experimental results measured by NMR velocimetry, although a close inspection of the profile at 
$z=1 \mathrm{~mm}$ next to the abrupt expansion indicates a lack of grid resolution for the 2 coarsest meshes only.

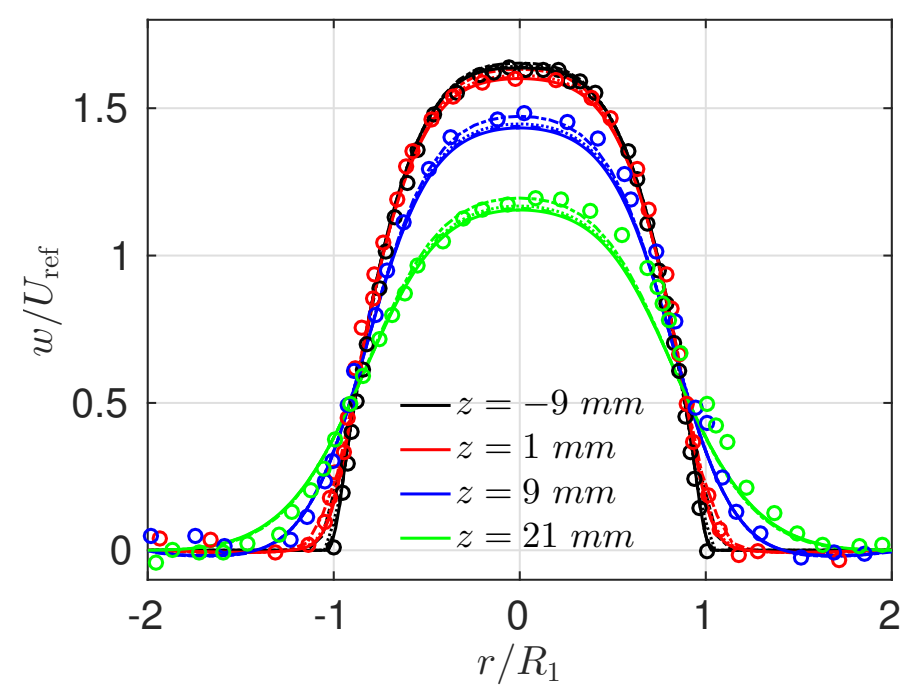

Figure 17: Grid convergence of axial velocity profiles at various cross sections before and after the expansion plane for the Xanthan $0.1 \%$ flow at $R e_{D_{1}}=56$. Circles (o) correspond to experimental results [1] while the dash-dotted (---), dotted (..), dashed (--) and solid (-) lines represent the LS-STAG solutions on meshes M1 to M4 respectively.

The main feature of expansion flows is the presence of a recirculating vortex caused by the separation of fluid flow at the singularity and its reattachment at the wall further downstream. For non-Newtonian fluids, the vortex characteristics in axisymmetric geometries have been studied experimentally in 28, 29] and numerically in 30, 31. The quantities of interest are the vortex length $L_{\mathrm{v}}$, its intensity $\Delta \psi$ characterized by the maximal value of the stream function in the recirculation zone, and the establishment length $L_{\mathrm{e}}$ which is the downstream distance to the expansion plane from which the axial velocity becomes constant. These quantities, non-dimensionalized with the step-height $d=\left(D_{2}-D_{1}\right) / 2$, are reported in Table 7 at $R e_{D_{1}}=56$ for meshes M1-M4. This table shows that nearly grid independent results are obtained on grid M3, with discrepancies below $1 \%$ for $L_{\mathrm{v}}$ and $L_{\mathrm{e}}$, and below $3 \%$ for $\Delta \psi$ compared to the M4 results. It can be observed that the higher is the shear-thinning index, the weaker is the intensity of vortex. In effect, since the drop in velocity gradients is more pronounced in the recirculation zone for high shear-thinning fluids, the bulk flow is able to drive the secondary flow at a lower rate. Furthermore, we observe that the establishment length increases significantly with the shear-thinning index, while the vortex size tends to shorten. A similar trend has also been observed in the numerical computations of [30] for power-law fluids and the experiments of [29] for purely viscous non-Newtonian fluids in the laminar regime.

More insight in the vortex characteristics is given by varying the Reynolds number, with computations performed at $R e_{D_{1}}=9,22,56,77.5,100,160$ and 217 on the M3 mesh. Figure 18 shows the vortex length predicted by the LS-STAG code and the axisymmetric FLUENT ${ }^{\circledR}$ computations of Rigal [11] with respect to the generalized Reynolds number 31] $R e_{\text {gen }}=2^{3-n}\left(\frac{n}{3 n+1}\right)^{n} R e_{D_{1}}$, where $n$ is the power-law index given in Table 5 As first observed by Halmos et al. [30, 28], the length of recirculation zone is a linear function of $R e_{\text {gen }}$ for all fluid types. Deviation from linearity for the FLUENT ${ }^{\complement}$ computations of the Xanthan $0.3 \%$ flows may be attributed to grid under-resolution [11. In addition, the empirical curve fitting of Nag \& Datta 31] have been added to this figure. For expansion ratio $\mathrm{ER}=2$, it predicts a linear growth of the recirculation zone as:

$$
\frac{L_{\mathrm{v}}}{\mathrm{d}}=\left(0.0297 n+0.0629 n^{2}\right) R e_{\mathrm{gen}} .
$$

However, the authors stated that this fitting is only valid for the range $R e_{\text {gen }} \in[50,200]$. It explains 
Table 7: Grid convergence study of the dimensionless vortex length $\left(L_{\mathrm{v}} / \mathrm{d}\right)$, vortex intensity $\Delta \psi^{*}=\Delta \psi /\left(\pi \mathrm{d}^{2} U_{\text {ref }}\right)$ and establishment length $\left(L_{\mathrm{e}} / \mathrm{d}\right)$ at $R e_{D_{1}}=56$. The establishment length is defined as the location where the axial and outlet velocity difference becomes lower than $2 \%$.

\begin{tabular}{|c|c|c|c|c|}
\hline Fluid type & Mesh & $L_{\mathrm{v}} / \mathrm{d}$ & $\Delta \psi^{*} \times 10^{3}$ & $L_{\mathrm{e}} / \mathrm{d}$ \\
\hline \multirow{4}{*}{ water } & M1 & 5.05 & 15.86 & 14.33 \\
\cline { 2 - 5 } & M2 & 5.00 & 15.32 & 14.17 \\
\cline { 2 - 5 } & M3 & 4.97 & 15.23 & 14.11 \\
\cline { 2 - 5 } Xanthan 0.1\% & M4 & 4.96 & 15.11 & 14.08 \\
\hline & M1 & 4.25 & 6.24 & 15.33 \\
\cline { 2 - 5 } & M2 & 4.10 & 5.68 & 15.33 \\
\cline { 2 - 5 } & M3 & 4.04 & 5.55 & 15.56 \\
\cline { 2 - 5 } Xanthan 0.3\% & M4 & 4.00 & 5.43 & 15.58 \\
\hline & M1 & 4.37 & 2.98 & 21.00 \\
\cline { 2 - 5 } & M2 & 4.16 & 2.44 & 21.00 \\
\cline { 2 - 5 } & M3 & 4.08 & 2.27 & 21.22 \\
\cline { 2 - 5 } & M4 & 4.03 & 2.16 & 21.33 \\
\hline
\end{tabular}

why the agreement of our computational results is excellent for the lower Reynolds number, while a deviation from this fit is observed as the Reynolds number is increased, most notably for the Xanthan $0.3 \%$ computations at $R e_{D_{1}} \geq 56$ (which corresponds to $R e_{\text {gen }} \gtrsim 200.1$ ).
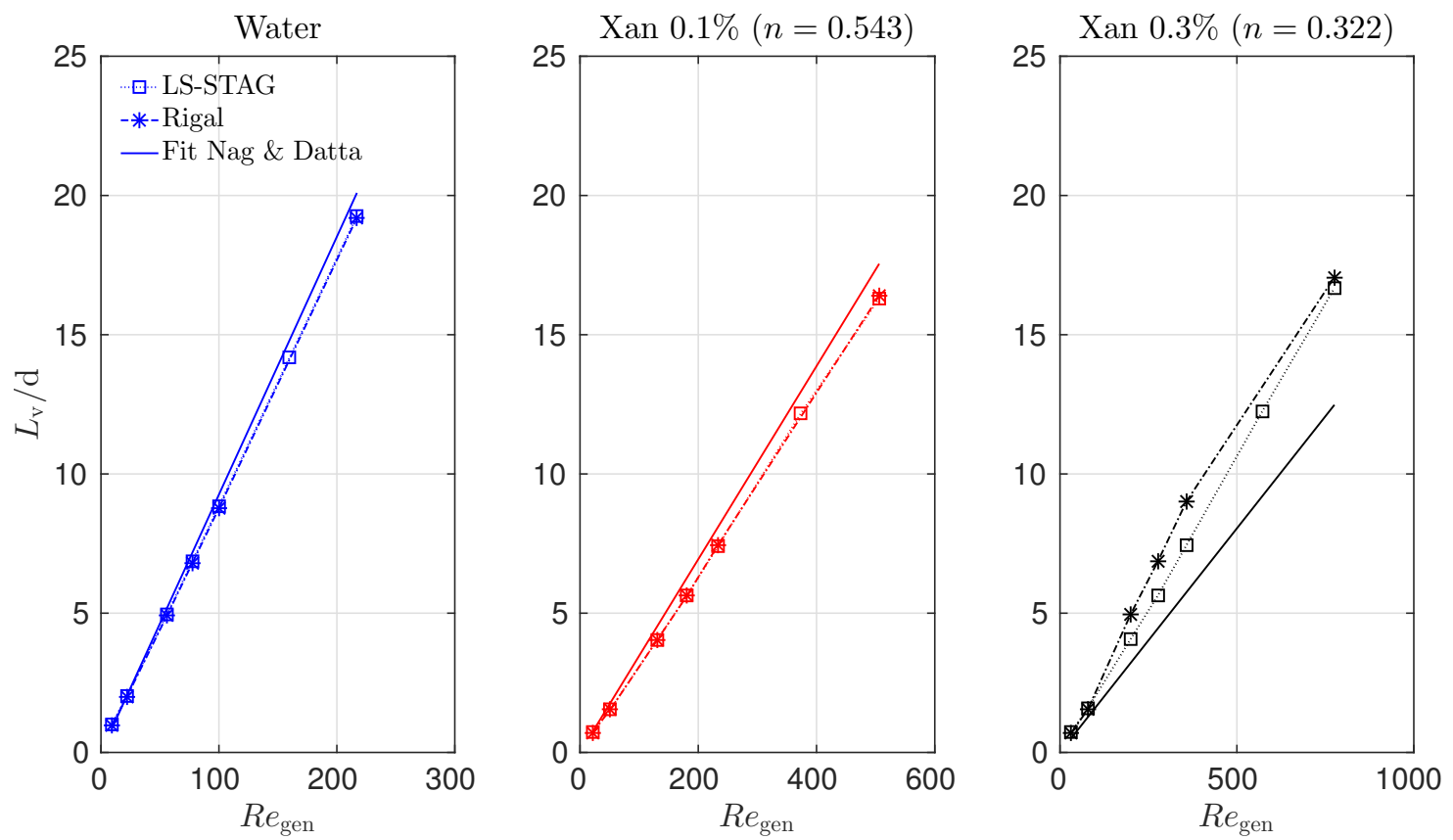

Figure 18: Variation of the vortex length with respect to the generalized Reynolds number.

Figure 19 displays the evolution of establishment length $L_{\mathrm{e}}$ and secondary vortex intensity $\Delta \psi$ with respect to the Reynolds number for the various fluids. The monotonic growth of $L_{\mathrm{e}}$ with the Reynolds number is directly related to the size of vortex. In effect, a longer recirculation zone allows the flow to decelerate more gradually, retarding thus flow establishment as the Reynolds number increases. Vortex intensity is a monotically increasing function of the Reynolds number that reaches a plateau at high Reynolds number. In [30, this behaviour is attributed to the increase in rate of momentum transfer from the bulk flow to the recirculation zone, until the momentum dissipated in the wall region reaches an asymptotical value. Similar trends were observed in [32, 33. 
for Newtonian flows with various expansion ratios.
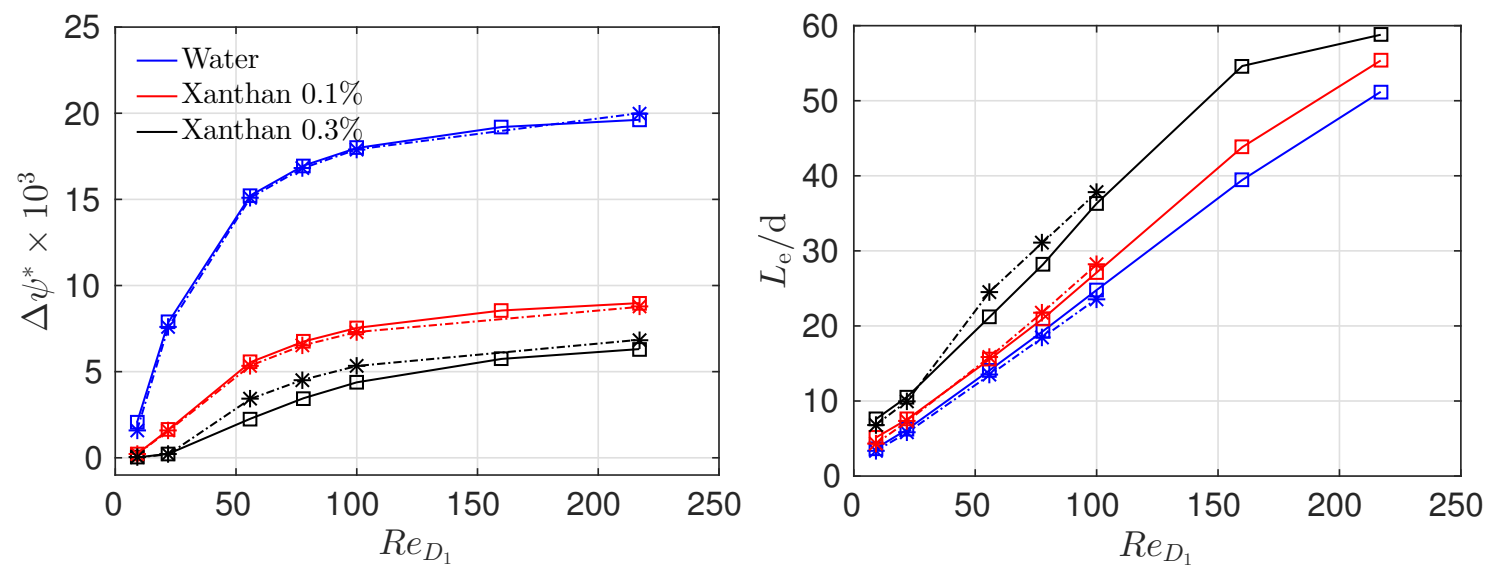

Figure 19: Evolution of vortex intensity (left) and establishment length (right) with the Reynolds number: (- $\square-$ ) LS-STAG and (-**-) FLUENT ${ }^{\circledR}[11$ results.

\section{Concluding remarks}

This paper has presented the extension of the LS-STAG cut-cell method to 3D extruded geometries in a parallel computing environment. A series of benchmark Newtonian and non-Newtonian flows have been conducted for assessing the performance of the LS-STAG solver (accuracy, computer resources) compared to well established flow solvers (Ansys-FLUENT ${ }^{\circledR}$ on body-fitted grids, immersed boundary method). To our knowledge, these issues have seldomly been addressed in the literature to such extent.

The results of these benchmark cases are very promising for cut-cell methods. The computations of the confined cylinder benchmark (Sect. 6.2.1) showed that a cut-cell solver was able to provide solution nearly as accurate as a commercial body-fitted CFD package, at a fraction of the computational cost. The DNS of turbulent cylinder flow (Sect. 6.2.2 highlighted the superior accuracy of the LS-STAG treatment of complex geometries compared to more traditional immersed boundary methods. Finally, the computation of Xanthan flows in axisymmetric sudden expansion illustrates the versatility of the method.

However, a close inspection on the accuracy of the LS-STAG method shows that the convergence rate is not as smooth as for methods on body-fitted grids. As stated in Sect. 6.1 and 6.2.1 this behaviour is certainly caused by the $a d-h o c$ discretization of the off-diagonal velocity gradients $\partial w / \partial x$ and $\partial w / \partial y$ in cut-cells, which is not yet as mature as the discretization of other terms in the Navier-Stokes equations. Future work will be dedicated to the improvement of the discretization of these terms, by adding non-orthogonal corrections in the fashion of the diamond scheme for unstructured grids [34, 16]. 


\section{References}

[1] R. Mittal, G. Iaccarino, Immersed boundary methods, Annu. Rev. Fluid Mech. 37, 239-261.

[2] S. Kang, G. Iaccarino, P. Moin, Accurate and efficient immersed-boundary interpolations for viscous flows, In Center for Turbulence Research Briefs, NASA Ames/Stanford University, pp. 31-43 (2004).

[3] Y. Cheny, O. Botella, The LS-STAG method : A new immersed boundary / level-set method for the computation of incompressible viscous flows in complex moving geometries with good conservation properties, J. Comput. Phys. 229, 1043-1076.

[4] O. Botella, M. Ait-Messaoud, A. Pertat, C. Rigal, Y. Cheny, The LS-STAG immersed boundary method for non-Newtonian flows in irregular geometries : Flow of shear-thinning liquids between eccentric rotating cylinders., Theoretical and Computational Fluid Dynamics 29, 93-110.

[5] O. Botella, Y. Cheny, F. Nikfarjam, M. Stoica, Application of the LS-STAG immersed boundary/cut-cell method to viscoelastic flow computations, Communications in Computational Physics 20 (2016) 870-901.

[6] P. van der Plas, H. van der Heiden, A. Veldman, R. Luppes, R. Verstappen, Efficiently simulating viscous flow effects by means of regularization turbulence modeling and local grid refinement, Seventh International Conference on Computational Fluid Dynamics (ICCFD7), Hawaii, paper ICCFD-2503, 2012.

[7] E. Bayraktar, O. Mierka, S. Turek, Benchmark computations of 3D laminar flow around a cylinder with CFX, OpenFOAM and FeatFlow, International Journal of Computational Science and Engineering 7 (3) (2012) 253-266. doi:10.1504/IJCSE.2012.048245

[8] R. Mittal, S. Balachandar, On the inclusion of three-dimensional effects in simulations of twodimensional bluff body wake flows, ASME Fluids Engineering Division Summer, Vancouver, British Columbia, Canada, 1997, available on CD-ROM only.

[9] A. G. Kravchenko, P. Moin, K. Shariff, B-spline method and zonal grids for simulations of complex turbulent flows, Journal of Computational Physics 151 (2) (1999) 757-789. doi: 10.1006/jcph.1999.6217.

[10] E. Balaras, Modeling complex boundaries using an external force field on fixed cartesian grids in large-eddy simulations, Computers and Fluids 33 (3) (2004) 375-404. doi:10.1016/ S0045-7930(03) 00058-6

[11] C. Rigal, Comportement de fluides complexes sous écoulement : Approche expérimentale par résonance magnétique nucléaire et techniques optiques et simulations numeriques, Ph.D. thesis, Université de Lorraine (2012).

[12] R. B. Bird, R. C. Armstrong, O. Hassager, Dynamics of Polymeric liquids, Wiley-Interscience, New-York, 1987.

[13] S. Osher, R. P. Fedkiw, Level Set Methods and Dynamic Implicit Surfaces, Springer, NewYork, 2003.

[14] R. W. C. P. Verstappen, A. E. P. Veldman, Symmetry-preserving discretization of turbulent flow, J. Comput. Phys. 187, 343-368.

[15] J. H. Ferziger, M. Peric̀, Computational Methods for Fluid Dynamics, Springer, Berlin, 1996.

[16] F. Moukalled, L. Mangani, M. Darwish, et al., The finite volume method in computational fluid dynamics, Springer, 2016, Ch. 8, pp. 211-270. 
[17] W. Gropp, E. Lusk, A. Skjellum, Using MPI: Portable Parallel Programming with the Message-Passing Interface, MIT press, 1999.

[18] R. Falgout, A. Cleary, J. Jones, E. Chow, V. Henson, C. Baldwin, P. Brown, P. Vassilevski, U. M. Yang, Center for Applied Scientific Computing (CASC), Lawrence Livermore National Laboratory. [link] URL http://acts.nersc.gov/hypre/

[19] M. Schäfer, S. Turek, F. Durst, E. Krause, R. Rannachar, Benchmark computations of laminar flow around a cylinder, in: E. H. Hirschel (Ed.), Notes on Numerical Fluid Mechanics (NNFM), Vol. 48, Vieweg+Teubner Verlag, 1996, Ch. Flow Simulation with HighPerformance Computers II, pp. 547-566. doi:10.1007/978-3-322-89849-4\{\_\}39.

[20] I. Orlanski, A simple boundary condition for unbounded hyperbolic flows, Journal of Computational Physics 21, 251 - 269.

[21] Ansys FLUENT theory guide,release 14.0 (2013).

[22] E. Bayraktar, Private communication (2016).

[23] C. H. K. Williamson, Vortex dynamics in the cylinder wake, Annual Review of Fluid Mechanics 28 (1) (1996) 477-539. doi:10.1146/annurev.fl.28.010196.002401.

[24] C. Norberg, Flow around a circular cylinder: Apects of fluctuating lift, Journal of Fluids and Structures 15 (3-4) (2001) 459-469. doi:10.1006/jfls.2000.0367.

[25] J. C. Hunt, A. Wray, P. Moin, Eddies, streams, and convergence zones in turbulent flows, Center for Turbulence Research, Proceedings of the 1988 Summer Program, 1988, pp. 193208.

[26] C. Wieselsberger, New data on the law of hydro and aerodynamic resistance, no. 84, NACA TN, 1992, (in German).

[27] B. Rajani, A. Kandasamy, S. Majumdar, Numerical simulation of laminar flow past a circular cylinder, Applied Mathematical Modelling 33 (2009) 1228-1247.

[28] A. Halmos, D. Boger, The behavior of a power-law fluid flowing through a sudden expansion. Part ii. experimental verification, AIChE Journal 21 (3) (1975) 550-553.

[29] B. Pak, Y. I. Cho, S. U. Choi, Separation and reattachment of non-newtonian fluid flows in a sudden expansion pipe, Journal of Non-Newtonian Fluid Mechanics 37 (2) (1990) 175-199.

[30] A. Halmos, D. Boger, A. Cabelli, The behavior of a power-law fluid flowing through a sudden expansion: Part i. a numerical solution, AIChE Journal 21 (3) (1975) 540-549.

[31] D. Nag, A. Datta, Variation of the recirculation length of newtonian and non-newtonian power-law fluids in laminar flow through a suddenly expanded axisymmetric geometry, Journal of Fluids Engineering 129 (2) (2007) 245-250.

[32] P. Scott, F. Mirza, J. Vlachopoulos, A finite element analysis of laminar flows through planar and axisymmetric abrupt expansions, Computers \& fluids 14 (4) (1986) 423-432.

[33] D. Badekas, D. Knight, Eddy correlations for laminar axisymmetric sudden expansion flows, Journal of fluids engineering 114 (1) (1992) 119-121.

[34] Y. Coudière, J.-P. Vila, P. Villedieu, Convergence rate of a finite volume scheme for a two dimensional convection-diffusion problem, ESAIM: Mathematical Modelling and Numerical Analysis 33 (3) (1999) 493-516. 